\title{
From Reactive to Endogenously Active Dynamical Conceptions of the Brain
}

\author{
Adele Abrahamsen \\ Center for Research in Language, University of California, San Diego \\ William Bechtel \\ Department of Philosophy, Center for Chronobiology, and Interdisciplinary Program \\ in Cognitive Science, University of California, San Diego
}

\begin{abstract}
We contrast reactive and endogenously active perspectives on brain activity. Both have been pursued continuously in neurophysiology laboratories since the early 20thcentury, but the endogenous perspective has received relatively little attention until recently. One of the many successes of the reactive perspective was the identification, in the second half of the $20^{\text {th }}$ century, of the distinctive contributions of different brain regions involved in visual processing. The recent prominence of the endogenous perspective is due to new findings of ongoing oscillatory activity in the brain at a wide range of time scales, exploiting such techniques as single-cell recording, EEG, and fMRI. We recount some of the evidence pointing to ways in which this endogenous activity is relevant to cognition and behavior. Our major objective is to consider certain implications of the contrast between the reactive and endogenous perspectives. In particular, we relate these perspectives to two different characterizations of explanation in the new mechanistic philosophy of science. In a basic mechanistic explanation, the operations of a mechanism are characterized qualitatively and as functioning sequentially until a terminating condition is realized. In contrast, a dynamic mechanistic explanation allows for non-sequential organization and emphasizes quantitative modeling of the mechanisms's behavior. For example, with appropriate parameter values a set of differential equations can be used to demonstrate ongoing oscillations in a system organized with feedback loops. We conclude that the basic conception of mechanistic explanation is adequate for reactive accounts of brain activity, but dynamical accounts are required to explain sustained, endogenous activity.
\end{abstract}


Observe a living organism — from a bacterium to a fellow human being — and you see an endogenously active system. Introspect and you will observe, as did William James, a continual flow of thoughts. If pressed, most neuroscientists and psychologists will acknowledge that neural systems are endogenously active, generating activity even in the absence of any stimulus. But for decades they have tended to disregard this key characteristic, pursuing programs of research in which they present discrete stimuli in structured tasks designed to focus on the neural and behavioral effects of experimental manipulations. In this paper we contrast this perspective (which we call reactive) with a dynamic perspective emphasizing endogenous activity. In neuroscience these have a long history of coexistence, but only recently has the endogenous perspective become less isolated as powerful new strategies for pursuing it have begun changing the overall research landscape. We provide a selective tour of this history from the vantage point of the new mechanistic philosophy of science, in which we highlight the interplay between basic and dynamic mechanistic explanation.

The reactive perspective has deep historical roots and is widely pursued in both neuroscience and psychology. Neuroscientists, following a tradition initiated by the British neurophysiologist Charles Scott Sherrington (1923), commonly treat the brain as a reactive mechanism in which sensory input initiates processing along a neural pathway, terminating in a motor response. One of their core techniques is to present stimuli and record neural responses in the brain area of interest; another is to manipulate neural activity and record motor responses. Psychologists more often target the whole organism, presenting stimuli and recording behavioral responses without determining the intervening neural activity. Most North American psychologists treated the gap between stimulus and response as a black box during the behaviorist era and as information processing thereafter, but for a minority (yesterday's psychobiologists and today's cognitive neuroscientists) the gap is filled by neural activity that should be investigated.

There is no doubt that this reactive framework has been enormously productive for both neuroscience and psychology. It has served to identify many of the parts and operations within the mechanisms responsible for cognitive phenomena, as we will show in the case of vision in section 2 . But there are also indications of its limitations. One is the considerable variability 
researchers commonly observe in both behavioral and neural responses. While this variability tends to be construed as noise to be eliminated from experimental data by averaging across time and subjects, if examined rather than concealed it can reveal compelling signatures of endogenous activity.

Laboratory research on endogenous activity, while relatively sparse, has historical roots nearly as deep as those of the reactive approach. Most notably, Thomas Graham Brown (1914) studied neural mechanisms for motor behavior in decerebrate cats alongside Sherrington in his laboratory at Liverpool from 1910 to 1913 — but arrived at quite different conclusions. Sherrington was commited to a sequential reflex mechanism, by which peripheral input (e.g., to the cat's feet when placed on a moving treadmill) produced a sequence of neural signals (to the spine, within the spine, and out to flexor and then extensor muscles). Each cycle of stepping resulted in renewed input (sensory feedback) and hence ongoing, rhythmic stepping movements. Brown discovered that he could obtain similar rhythmic stepping even after isolating the spinal cord from afferent (peripheral) input by cutting the dorsal root nerves. This impressive demonstration of endogenous control led him to propose a neural mechanism that later would be recognized as the first description of a central pattern generator - central because the key components were in the spine (sensitive to but not dependent on peripheral input); pattern because it produced an ongoing oscillatory pattern (observed as rhythmic stepping, in which flexion alternates with extension); and generator because this mechanism could initiate production of the pattern. More specifically, Brown proposed what would now be described as two coupled networks of spinal neurons - one for flexion and one for extension—which oscillated in inhibiting each other's activity. However, Sherrington resisted distraction from his own pursuit of a sequential reflex account of motor behavior; ${ }^{1}$ as his reactive approach became more entrenched, Brown was increasingly marginalized (for discussion, see Stuart \& Hultborn, 2008).

It was a half-century before Brown's emphasis on endogenous activity was revived by researchers who converged on central pattern generators as the explanatory mechanism of choice

\footnotetext{
${ }^{1}$ Early on Sherrington (1913, p. 207) acknowledged that Brown's view "demands careful attention" but demurred on grounds that his own line of explanation "would be led too far afield by its consideration now."
} 
for a variety of rhythmic motor behaviors. Wilson and Wyman's (1965) landmark account of flight in locusts was followed by identification of central pattern generators in the brain stem and spinal cord for such activities as walking, swimming, respiration, and circulation (Grillner, 2003). Almost another half-century passed before neuroscientists investigating sensory processing and central cognition turned their attention to endogenous activity in cerebral cortex and were rewarded with multiple streams of evidence from single cell recording, EEG, fMRI, and other techniques. We introduce some of this evidence in section 3 , and emphasize that the resulting conception of the brain as endogenously active poses a profound challenge to the purely reactive perspective that has dominated much of psychology as well as neuroscience.

The slow spread of the endogenous perspective is unsurprising considering the history of other sciences. Max Planck (1949, pp. 33-34) famously suggested that "A new scientific truth does not triumph by convincing its opponents . . but rather because its opponents eventually die." He exaggerated for effect, presumably, but it is not uncommon for scientists to bemoan delays in the uptake of new approaches. Less remarked upon is the delayed impact of changes in the sciences on philosophy of science. This is a young field (its first journal, Philosophy of Science, began publication in 1934), and it has been slow to move beyond its initial roots in twentieth-century physics to incorporate quite different influences from the biological and cognitive sciences. We suggest that this delay has been excessive and detrimental to its own development as a field of inquiry. Philosophers of science did not even recognize the dominant mode of explanation in these sciences — mechanistic explanation — until pioneering work by William Wimsatt, who pointed out that "At least in biology, most scientists see their work as explaining types of phenomena by discovering mechanisms ...” (Wimsatt, 1976, p. 671). His influence on a cohort of students gave rise in the 1990s and especially after 2000 to the new mechanists, who have drawn on biology and cognitive science rather than physics in constructing a new mechanistic philosophy of science (Bechtel \& Richardson, 1993/2010; Bechtel \& Abrahamsen, 2005; Glennan, 1996, 2002; Machamer et al., 2000; Thagard, 2003; Wimsatt, 2007).

Recently we have argued that further developments in these sciences-especially computational modeling of the dynamics of cognitive and neural mechanisms - require extending the mechanistic framework to incorporate dynamic mechanistic explanation (Bechtel \& 
Abrahamsen, 2010, 2011). Thus, in what follows we begin by distinguishing between basic mechanistic explanation, in which target systems are treated as reactive mechanisms, and dynamic mechanistic explanation, which has the resources to characterize endogenous as well as reactive activity and to do so with greater precision (section 1). We then discuss investigations of brain mechanisms in particular, contrasting those that exemplify the reactive perspective (section 2 ) with those targeting endogenous activity (section 3 ). We consider certain implications of the endogenous perspective for how we understand cognitive activity (section 4). Finally, we return to the philosophical understanding of dynamic mechanistic explanation and how it can illuminate research that takes an endogenous perspective on the brain (section 5).

\section{Two Conceptions of Mechanism}

The new mechanists have primarily focused on basic mechanistic explanation, in which investigators decompose a system into a set of component parts, each of which performs one or more operations, and recompose it by figuring out the spatial organization of the parts and temporal/causal organization of the operations (Bechtel \& Abrahamsen, 2005, 2009). The idea is that going down to a lower level provides the most useful explanation of how the system's activity generates a phenomenon of interest.

What makes these explanations "basic" is that the accounts of organization are mostly qualitative rather than quantitative. Thus, a typical structural decomposition into parts would be recomposed into a spatial ordering (e.g., the spine's lumbar vertebrae are designated as L1, L2, L3, L4, L5) or a schematic layout (e.g., a eukaryotic cell is depicted as a membrane enclosing one nucleus and numerous organelles in cytoplasm). A typical functional decomposition into operations would be recomposed most simply into a temporal ordering in which the product of one operation is operated upon by the next (e.g., the chain of biochemical reactions comprising intermediary metabolism). The act of constructing a basic mechanistic explanation of a phenomenon is complete $^{2}$ when the investigator can specify which parts perform which operations. This task of

\footnotetext{
2 "Complete" does not imply "final." An important role for such an account is to provide a framework for further research that elaborates and corrects it and eventually may replace it. Darden and Craver (2002) referred to incomplete accounts as mechanism sketches and traced how two different sketches for protein synthesis in the 1950s
} 
localization sometimes is integral to the discovery process, but may instead be deferred (pending development of necessary tools, for example, the electron microscope). Once achieved, a wellsupported basic mechanistic account is an important research milestone.

One example, discussed at greater length in section 2, is a pathway through the visual system that is responsible for the phenomenon of object recognition. Parts of the pathway have been identified in the retina, lateral geniculate nucleus (LGN), occipital lobe (visual areas V1, V2, and V4) and temporal lobe. A very simplified version of the basic mechanistic account has each of these parts in turn performing one or more operations on the output of the preceding operation: ${ }^{3}$ the retina represents a stimulus object topographically, the LGN modulates or gates the representation, V1 extracts several types of features, V2 analyzes contours, V4 analyzes form and color, and inferior temporal cortex performs higher-level, integrative operations that yield a percept recognized as a particular type of object.

It is possible to find ordered components with no beginning or end: the beads in a bracelet, the bases in loops of mitochondrial DNA, people circle-dancing, and so forth. But something in us likes an ordering to be not only invariant but also bounded and unidirectional. Scientists are no exception, showing a preference for basic mechanistic accounts in which operations are ordered with a beginning and an end. We will reserve the term sequence for this type of organization in time or space.

Sequential organization is especially prominent in the definition of mechanism offered by Machamer, Darden, and Craver (2000):

Mechanisms are entities and activities organized such that they are productive of regular changes from start or set-up to finish or termination conditions.

Note that their terms "entities" and "activities" are equivalent to parts and operations respectively. (We use the term "activity" to refer to the overall behavior of a mechanism as distinguished from the component operations.)

were gradually modified and brought together in a basic mechanistic account that was completed (but not final) in the 1960s.

${ }^{3}$ The outputs of operations arguably are (a special class of) parts. This is clearer in the case of biochemical reaction pathways, in which the outputs are molecules, than in the case of neural pathways, in which the most useful characterization of the output often is an abstract representation. 
What we wish to highlight here is their explicit stipulation of a beginning and end. In the case of protein synthesis, as discussed by Darden and Craver (2002), this seems appropriate. The start or set-up conditions are not itemized, but they would seem to include the availability of ribosomes and amino acids in the cytoplasm, the availability of several kinds of RNA where needed, and (crucial to initiation of the process) the appropriate RNA polymerase coming into proximity with the DNA segment that codes for the protein. Highlights of the "regular changes" (sequence of operations) enabled by those conditions include the RNA polymerase unzipping and transcribing the DNA into a complementary mRNA base sequence, the transport of the mRNA into the cytoplasm, each codon (sequence of three bases) on the mRNA forming a weak hydrogen bond with an appropriate tRNA, guiding its attached amino acid to form a peptide bond with the previous tRNA's amino acid. These last two operations are repeated for each codon in turn, hence synthesizing the protein one amino acid at a time. When the last peptide bond has been formed, the key termination condition of the protein synthesis mechanism has been satisfied and it stops.

This case and the definition itself exemplify the reactive perspective, insofar as a sequence of activity is initiated by satisfaction of set-up conditions and ends with satisfaction of termination conditions. Many cases in biology are less good exemplars. Machamer, Darden and Craver (2000, p. 11) acknowledged that set-up conditions "may be the result of prior processes" but justified requiring them on grounds that "scientists typically idealize them into static time slices taken as the beginning of the mechanism." They further noted that "the bulk of the features in the set-up ... are not inputs into the mechanism but are parts of the mechanism." A focus on internal components is indeed a strength of any mechanistic account, as contrasted with purely functional accounts of input-output relations. Nonetheless, set-up and termination conditions misleadingly suggest that the system targeted for explanation is passively awaiting initiation of activity that, once underway, reaches a stopping point. Since biological mechanisms typically function continually, what investigators have treated as start-up conditions are better viewed as perturbations to ongoing endogenous activity. 
To build a mechanism capable of sustained, endogenous activity a minimal first step is to allow at least one operation posited as later in the sequence to feed back on operations posited as earlier. Adding even a single negative feedback loop to an otherwise feedforward mechanism can produce ongoing dynamic activity, most notably oscillations. In a mechanism with appropriately weighted feedback and openness to energy, these oscillations can be regular (exhibiting, for example, a stable frequency of $10 \mathrm{~Hz}: 10$ cycles of rise and fall per second) and self-sustained (i.e., not dampen to a steady state over time; see Goodwin, 1965). Many actual biological systems are well-characterized by a mechanistic account in which positive as well as negative feedback loops are added to a sequential backbone of operations. Carbohydrate metabolism, for example, is achieved by a chain of reactions that begins with glycogen and ends with pyruvate. At least in vitro, sideloops regulate the system such that the amount of pyruvate produced oscillates with a frequency of about one cycle per minute. (Examples from metabolism are further discussed in Bechtel \& Abrahamsen, 2011). It should be note that this glycolytic oscillator is harmonic - the amount of pyruvate changes at a constant rate. Neural oscillators, in contrast, are relaxation oscillators - also regular, but with pulselike activity (spikes) against a low-activity background.

The addition of feedback loops is not a trivial adjustment: it is a key means of moving beyond a purely sequential conception of mechanism to a more dynamic conception. Our own earlier characterization of mechanism gestured in the direction of dynamics in referring to "orchestrated functioning of the mechanism," but we recently augmented it as follows:

A mechanism is a structure performing a function in virtue of its component parts, component operations, and their organization. The orchestrated functioning of the mechanism, manifested in patterns of change over time in properties of its parts and

operations, is responsible for one or more phenomena. (Bechtel \& Abrahamsen, 2010). The phrase in boldface was added to explicitly cover a broader range of mechanistic accounts offered by scientists: not only sequential accounts but also those in which the parts and operations are organized so as to generate endogenous oscillations or other interesting dynamics. This led us directly to consideration of how the dynamics might be characterized. Those scientists who emphasize laboratory research tend to look first to their data for this, as in the example above of pyruvate concentrations oscillating at about one cycle per minute. They then 
attend to what operations and organization might be responsible for the dynamics observed. (In this example, they were able to show that the feedback loops involving one particular enzyme early in the reaction pathway were crucial.). Another approach is important as well. A computational biologist can use mathematical tools to construct a computational model that is explicitly grounded in a mechanistic account. The model offers a precise (and potentially falsifiable) characterization of the mechanism's dynamics. The variables in the model are more or less directly aligned with properties of parts and operations in the mechanistic account. ${ }^{4} \mathrm{~A}$ computational modeler can capture various oscillatory patterns produced by biological mechanisms and then determine whether there are realistic values of the model's parameters for which the oscillations are self-sustaining. This is of particular interest when endogenous oscillations are claimed. In brief, dynamic mechanistic explanation encompasses both laboratorybased and computational research. Ideally (but not usually) these are carried out collaboratively.

Endogenously active mechanisms typically can be affected by exogenous inputs, but how they respond to these depends upon their current endogenous state, which may vary systematically or irregularly over time. It is important to understand the underlying endogenous behavior of the mechanism in order to understand how it responds to perturbations. The situation becomes even more important when the endogenous behavior of one mechanism is affected by endogenous activity in other mechanisms with which it is dynamically linked (e.g., within an organism's body, or within an ecological network in which the organism is behaving). We return to the discussion of how understanding endogenous activity is relevant to understanding the responses of mechanisms to exogenous inputs in section 4 .

\section{Traditional Experimental Approach to the Brain}

Although we will focus on shortcomings of the reactive conception of mechanism, research programs grounded in that conception have been enormously productive. Indeed, researchers inclined to dynamic mechanistic accounts typically are not in a position to advance serious

\footnotetext{
${ }^{4}$ Most simply there is direct correspondence between variables and properties; for example, $c$ may denote the concentration of the product of a reaction and $r$ the rate of the reaction. Sometimes, though, it is a more complex expression in the model (e.g., a variable multiplied by a scaling parameter) that corresponds to a property in the mechanistic account (e.g., the rate of a reaction).
} 
proposals about the integrated, dynamical behavior of a mechanism until researchers pursuing the reactive approach have provided a rich understanding of the parts and operations within it. Further, a premature emphasis on the whole integrated system can be counterproductive. Brain research in the $18^{\text {th }}-20^{\text {th }}$ centuries was marked by ongoing tension between mechanists who sought to localize specific mental functions in specific areas of cerebral cortex and holists who argued that the activity required for particular functions was broadly distributed. For example, Ferrier's (1876) localization of a number of sensory and motor functions based on ablation and stimulation experiments in monkeys were countered in the 1880s by Goltz's claim that such functions were preserved in dogs with extensive ablations. Post-mortem examinations supported Ferrier, and some degree of localization of sensory and motor functions became widely accepted (see Finger, 1994, pp. 54-56). The debate continued with respect to localization of intellectional functions, such as memory and reasoning, in remaining areas of cerebral cortex. The claim that what matters is the amount of tissue destroyed in ablations, not which tissue, received its bestknown expression in Lashley's (1929) "law of mass action" and goes back at least to Flourens (1824). But whatever its merits, this holistic view that large parts of cortex act as a distributed, integrated system did not generate a positive program of inquiry. History has adjudicated that it was the researchers pursuing localization of functions in the brain who achieved results that could be built upon, leading to our current mechanistic accounts of how the brain performs cognitive tasks.

The reactive perspective on the brain is well exemplified in research on visual processing. In the late $19^{\text {th }}$ century it was established that a key area for visual processing was a region of the occipital lobe distinguished by its pattern of striation (hence, striate cortex; now called VI). Neural pathways were traced from the eyes to this region, and lesions to it produced visual deficits in both humans and animals. Salomen Henschen (1893) determined that damage to particular regions within striate cortex resulted in blindness to specific parts of the visual field, leading him to propose that striate cortex was organized in terms of a topographic map of the visual field. While the idea of topographic maps has endured, the particular map Henschen proposed turned out to be inverted from the ones subsequently developed by Tatsuji Inouye (1909) and Gordon Holmes (1918) based on correlations between visual deficits and brain damage in soldiers (Figure 1). 

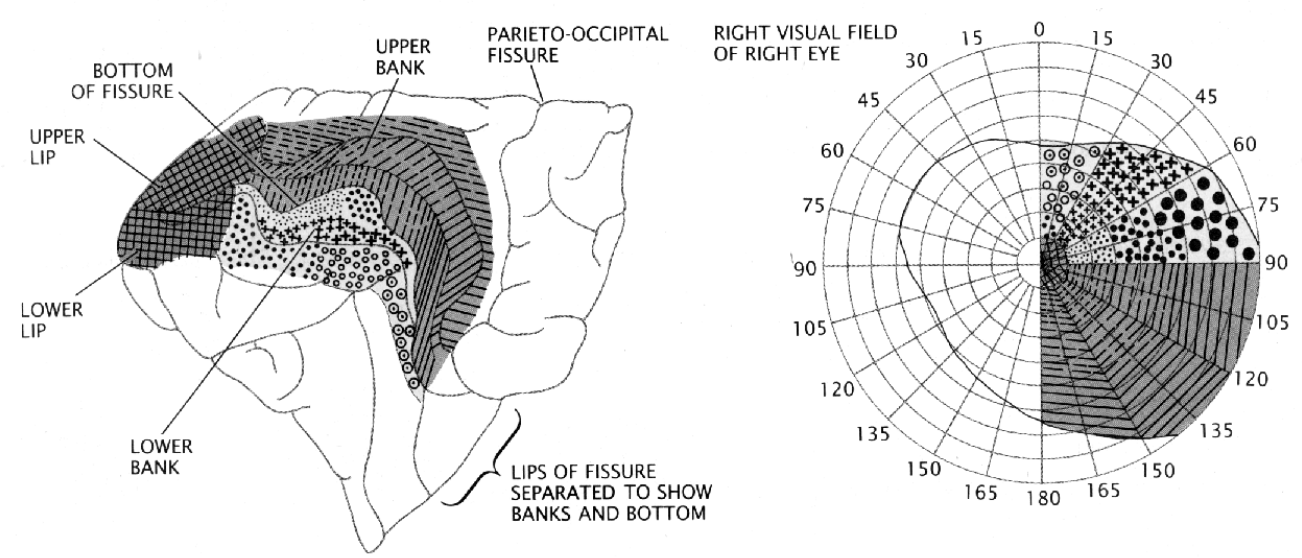

Figure 1. Goldon Holmes' (1918) map indicating how different areas of the right visual field (the right side of the image on the right) project onto particular regions of the left occipital lobe (shown on the left).

Later in the $20^{\text {th }}$ century, electrophysiologists developed techniques for recording activity from individual neurons in response to carefully selected visual stimuli. Single-cell recording enabled researchers to determine not only where each neuron was responsive (yielding much finergrained topographic maps) but also how it responded. It turned out that the topographic mapping strategy was relied upon in multiple regions - retina, LGN, striate cortex, and beyond - but that none produced a simple pixel-like representation. Each region had its own distinctive computations awaiting discovery. In pioneering work, Kuffler (1953) found that retinal ganglion cells in cats respond best to light spots on dark backgrounds or dark spots on light backgrounds. He proposed that these center-surround cells processed stimulation at the center of their receptive fields and stimulation at the immediately surrounding area in an antagonistic manner (responding maximally if one was dark and the other light).

Hubel and Wiesel demonstrated that the center-surround design is replicated in cats' LGN (the lateral geniculate nucleus of the thalamus), but failed to find it in striate cortex. The edge of a misoriented slide sparked their realization that striate neurons respond not to spots but to linear stimuli (light or dark bars or edges). They proceeded to differentiate simple cells (those responsive to a bar at a specific locus and orientation) from complex cells (which respond to bars anywhere within a broader area of the visual field but especially to those moving in a preferred direction). As illustrated in Figure 2, Hubel and Wiesel (1962) proposed that a bar in the visual 
field spanned the receptive fields of several center-surround cells in the LGN. These were connected to at least one simple cell that detected their joint activation, and multiple simple cells (ideally with closely adjacent receptive fields) in turn were connected to each complex cell. A complex cell was especially responsive if the simple cells connected to it were triggered in sequence by a bar moving in the appropriate direction. The simple and complex cells can be thought of as engaging in feature detection at two levels. In a subsequent study Hubel and Wiesel replicated these findings of simple and complex cells in monkeys but also reached the conclusion that their activity "represented a very elementary stage in the handling of complex forms" and must be followed by further processing "at later stages in the visual path" (Hubel \& Wiesel, 1968, p. 242). They began referring to striate cortex as V1 (visual area 1) and the areas involved in later stages of processing as V2, V3, V4 and MT (medial temporal area) —clearly embracing the conception of the visual system as a sequential processor, with its sequence of operations initiated by presentation of a visual stimulus and culminating in a percept.

(a)

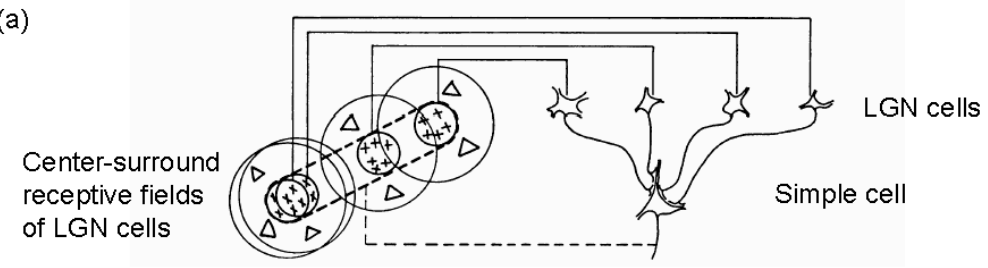

(b)



Figure 2. Hubel and Wiesel's (1962) proposed simple and complex cells in striate cortex. (a) Center-surround cells in LGN that detect spots are connected to simple cells that detect locationand orientation-specific bars. (b) Simple cells are connected to complex cells that detect orientation-specific bars, especially those moving in a particular direction.

Hubel and Wiesel's strategy found numerous applications in subsequent years as researchers inferred function from the classes of visual stimuli that drove responses in specific regions of occipital, temporal, and parietal cortex. For example, neurons in area V4 were found to achieve color constancy: in addition to responding to variations in the incoming wavelength due to 
changes in the color of an object (like V1) they compensate for variations due to changes in its illumination. Similarly, neurons in area MT were discovered to respond to the perceived direction of movement of complex stimuli, whereas those in V1 presented with the same stimuli respond only to the direction in which components of the stimuli move across the visual field. Subsequent research revealed regions in the temporal lobe that respond to specific classes of objects and regions in the parietal lobe that respond to their spatial location (each with distinct pathways from subareas of LGN, V1 and so forth). By the 1990s over thirty different brain areas in the macaque had been identified as engaged in processing visual stimuli, and for many of these areas research pursuing the approach just described succeeded in determining the specific features of stimuli that evoked a response (van Essen \& Gallant, 1994). Each successive brain area was regarded as operating on the products generated in previous areas to extract new information about the visual stimulus.

By any measure, this research endeavor that treated the visual system as reacting to visual stimuli was extremely successful (for a detailed account of this century of research, see Bechtel, 2008). There are reasons to suspect, however, that the resulting explanatory accounts may be incomplete and require non-trivial revision. First, the approach assumes sequential processing of inputs by a succession of processing centers. But researchers have long known that in addition to forward axonal projections there are extensive backwards and collateral projections in this system (Lorente de Nó, 1938). Hubel and Wiesel had found that the neurons they recorded were organized into columns traversing the six layers of cortex and that neurons within a column responded to stimuli in the same part of the visual field. Forward, backwards, and collateral projections could be differentiated by the layers from which and to which they projected, which helped researchers uncover the complex pattern of connections between visual areas in the macaque. The findings were displayed visually in the well-known "subway map" diagram by Felleman and van Essen (1991), as shown in Figure 3. The other indication that sequentiallybased accounts require revision is that researchers have constantly confronted the problem that neural responses are highly variable. This variability is generally regarded as noise which needs to be removed so as to reveal the signal - but a very different research program emerges if instead it is taken to indicate that much more may be going on within the mechanism than is revealed by what is regarded as signal. 


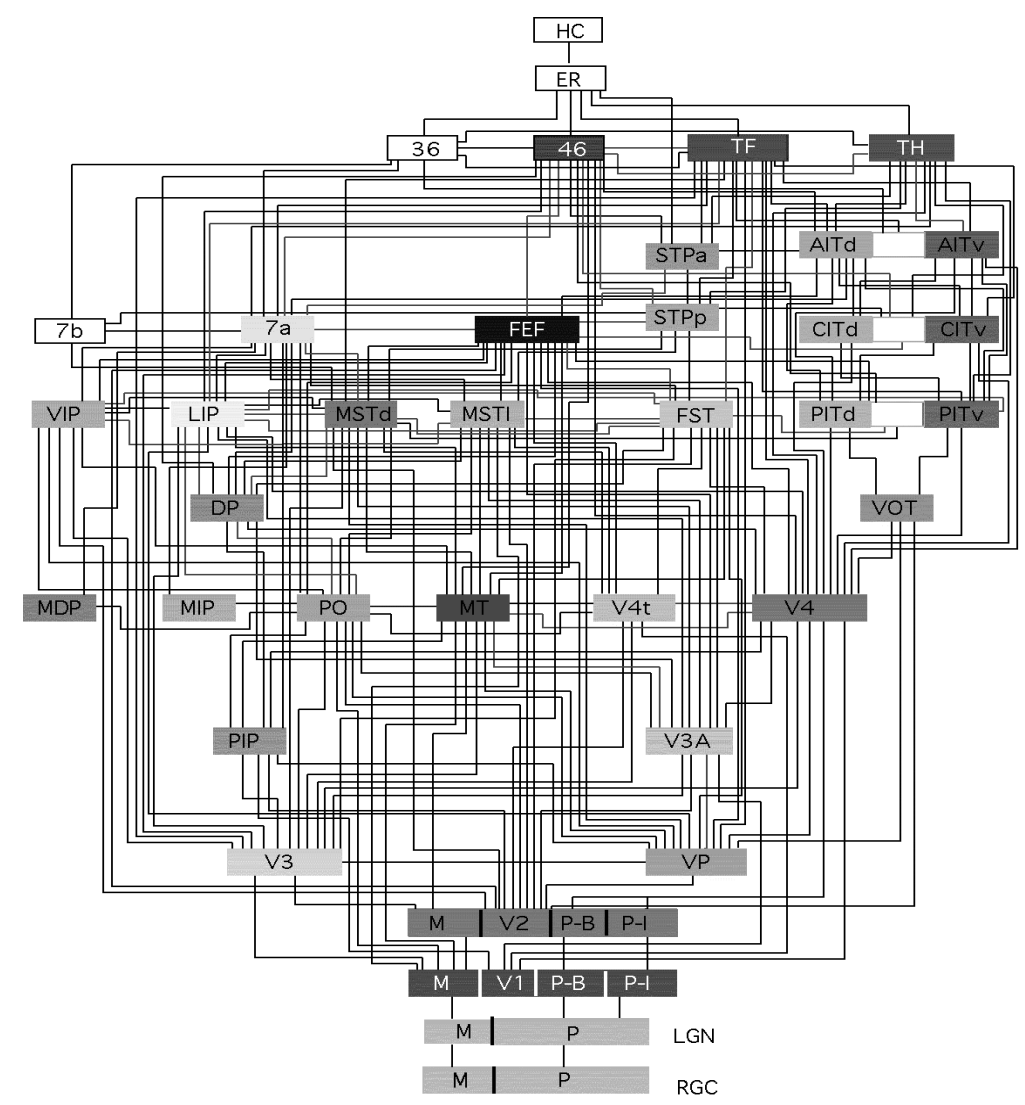

Figure 3. Felleman and van Essen's (1991) representation of 32 cortical visual areas identified in the macaque and the known connections between them. In most cases the connections are bidirectional, with separate bundles of axons running between different layers for feedforward vs. feedback signals (not shown).

\section{Reconceptualizing the Brain as Endogenously Active}

The alternative conception of the brain as an endogenously active mechanism is being pursued by a growing vanguard of neuroscientists. They are rethinking brain dynamics, are redirecting the tools of their trade towards detection of endogenous activity, and are devising analyses that can describe that activity and tease out interactions with activity evoked by stimuli. We will discuss three key technologies in the order in which they began to be directed to uncovering endogenous activity in the brain. Since they differ in their temporal and spatial range and resolution, we conclude this section by asking how activities at multiple timescales might interrelate. 


\section{a. Electroencephalography (EEG)}

The vision researchers discussed above implanted electrodes so as to record the activity of individual neurons, but there is an even longer tradition of inferring aggregate activity from electrodes placed on or into the scalp or (in animals or surgical patients) on the cortical surface. The difference in electrical potential between two electrodes fluctuates over time, providing a measure of electric currents in the brain with high temporal but low spatial resolution. In pioneering research with rabbits and monkeys, Richard Caton (1875) experimented with various placements of pairs of electrodes connected to a mirror galvanometer that represented the currents visually. Despite primitive tools, he made the first observations both of continuous spontaneous activity ("feeble currents") and of localized "negative variation" evoked by a stimulus. $^{5}$

Caton's technique was reinvented more than once, but did not give rise to an ongoing program of research until psychiatrist Hans Berger adapted it to humans in the 1920s. Berger initially inserted needle electrodes into subcutaneous tissue, often one at the front and one at the back of the head, but found that he could obtain similar results with the less intrusive procedure of affixing lead foil electrodes to the scalp. With electrodes connected to a string or double-coil galvanometer that was attached to a recording apparatus, he could permanently capture oscillations in the current as lines on long strips of paper (with some delay since the recording involved a photographic process). In his first publication (Berger, 1929), he called this an Elektrenkephalogramm or, in English, an electroencephalogram (EEG), in recognition of existing electrocardiogram instrumentation which he had adapted. In patients and healthy individuals at rest with eyes closed, he repeatedly observed two distinct waveforms. In his next report (Berger, 1930), he coined the term alpha waves for the approximately $10 \mathrm{~Hz}$ oscillations that most intrigued him and the term beta waves for smaller, faster $20-30 \mathrm{~Hz}$ oscillations. Moreover, Berger discovered what was later called alpha blocking: when the eyes were opened

\footnotetext{
${ }^{5}$ One of Caton's objectives was to evaluate Ferrier's claims regarding localization of motor commands, and he reports (p. 278): “on the areas shown by Dr. Ferrier to be related to rotation of the head and to mastication, negative variation of the current was observed to occur whenever those two acts respectively were performed. Impressions through the senses were found to influence the currents of certain areas; e. g., the currents of that part of the rabbit's brain which Dr. Ferrier has shown to be related to movements of the eyelids, were found to be markedly influenced by stimulation of the opposite retina by light."
} 
alpha waves declined precipitously, leaving beta waves to predominate. Even with eyes closed, events in other sensory modalities or attention-demanding tasks such as mental arithmetic could produce this effect. For example, Figure 4 shows the "striking change" from predominantly alpha to beta waves that Berger obtained by stroking his subject's hand with a glass rod.

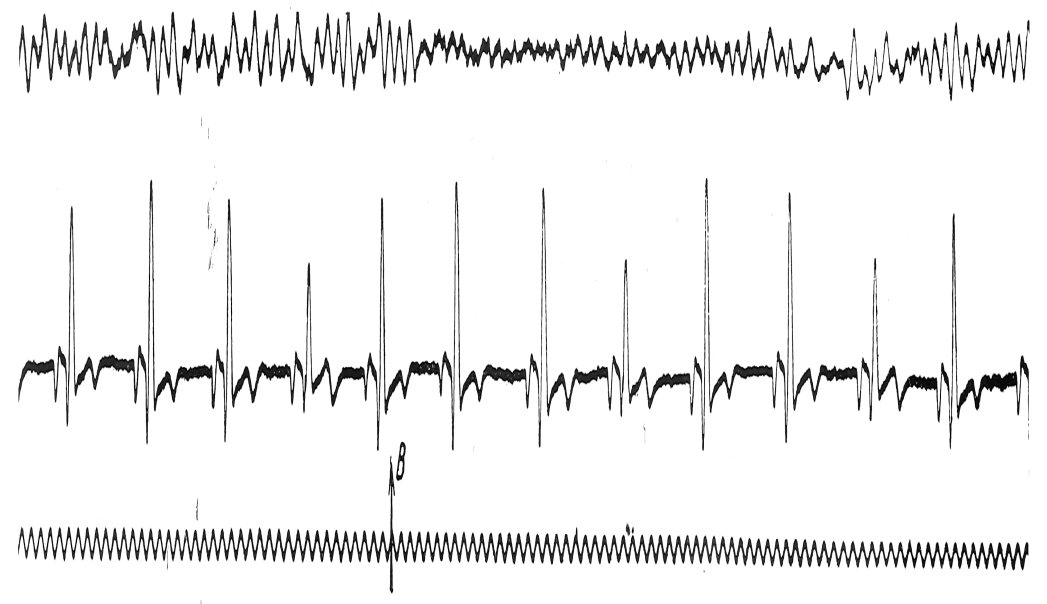

Figure 4. In this eight-second extract of a recording made by Berger (1930), the upper line is a subcutaneous EEG. It shows three seconds of predominantly alpha waves that were blocked 0.27 seconds after he stroked the subject's hand with a glass rod (indicated by arrow "B" on the $10 \mathrm{~Hz}$ timing signal at the bottom). For at least the next two seconds the EEG shows lower-amplitude, higher-frequency beta waves, not alpha waves. The middle line is an electrocardiogram recorded simultaneously. Extracted from Figure 5 in Gloor's translation (1969, p. 82) of Berger (1930).

Berger conducted extensive control studies to show that EEG oscillations were not due to artifacts but in fact provided a window on the brain's endogenous and evoked activity. (In Figure 4, for example, the EEG was not obviously correlated with the electrocardiogram displayed beneath it.) Few were convinced until he attracted the attention of Edgar Adrian, a leading investigator in neurophysiology at Cambridge University. Adrian and Matthews (1934) were initially skeptical, based on their own recordings in rabbits and cats, but found compelling evidence in humans of the alpha rhythm and attributed it (p. 384) to "the spontaneous [synchronous] beat of an area in the occipital cortex which is normally occupied by activities connected with pattern vision."

\footnotetext{
${ }^{6}$ Adrian and Matthews limited their focus to the alpha rhythm, which they called "the Berger rhythm," and characterized it as disappearing in task conditions. They further claimed that "a group of cortical cells in some part
} 
With Adrian's imprimatur, human EEG research attracted other pioneering investigators; within a decade, three additional rhythms had been investigated and named. The term gamma rhythm was proposed by Jasper and Andrews (1938) to designate frequencies above 30 or $35 \mathrm{~Hz}$, but high-quality evidence for functionally distinguishing gamma from beta rhythms is much more recent and often involves evoked rather than endogenous activity. (Proposals regarding functions of evoked gamma activity have included object perception, cross-modal perception, feature binding, aspects of short-term memory, and even - focusing more on endogenous than evoked activity - consciousness and ongoing information processing.) The other two rhythms involved slower waveforms that Berger had already associated with brain lesions and sleep. W. Grey Walter (1936), in reporting EEG studies of awake humans with brain tumors, proposed the term delta rhythm for waves lower in frequency (and typically higher in amplitude) than the alpha rhythm. Later he designed an automated frequency analyzer and, deploying it on EEGs from a variety of patients, differentiated a primarily subcortical theta rhythm $(4-7 \mathrm{~Hz})$ from the slower $(<4 \mathrm{~Hz}$ ), primarily cortical delta rhythm (Walter \& Dovey, 1944).

The subsequent years brought improvements in recording technologies (e.g., digital EEG in the 1960s) and in analysis of complex EEG waveforms. Methods generally proceed from the assumption that these waveforms can be decomposed into sinusoidal components of different frequencies. Even Berger had noted that irregularities in the alpha waves ("notches" on their descending limbs too small to see in Figure 4) indicated that they were always mixed with the smaller beta waves. However, automated analysis as pioneered by Walter is far more revealing than visual inspection of EEG recordings. Since the 1960s computers have allowed efficient calculation of power density (a measure of amplitude) for each frequency or frequency band within a time window. Current variations on this method use a fast Fourier or wavelet transform of the EEG waveform. Herrmann, Grigutsch, \& Busch (2005) provide an introduction to wavelet

of the occipital lobe ... tend to beat synchronously when they are undisturbed, but visual activity or widespread non-visual activity in the brain breaks up the rhythm by exposing the cells to a mosaic of excitations which makes synchronous action impossible. Berger, if we have interpreted him correctly, regards the waves as having a much wider and less specific origin, but the evidence as to localization is the only important point on which our results seem to differ from his" (p. 356). Later researchers confirmed a primary localization in the occipital lobe, but also found other origins and/or broader activity under some conditions. Others confirmed that, although alpha rhythms are not prominent in most animal species, Berger was correct in reporting that they were prominent in dogs as well as humans (see especially pp. 239 and 256 in the review by Shaw, 2003.) 
analysis and suggest the following as well-established frequency bands: delta (0-4 Hz), theta (4-8 $\mathrm{Hz})$, alpha $(8-12 \mathrm{~Hz})$, beta $(12-30 \mathrm{~Hz})$, gamma $(30-80 \mathrm{~Hz})$. Additional, less standardized bands are sometimes specified by those taking advantage of recent advances in technology; examples include infraslow $(0.01-0.1 \mathrm{~Hz})$, very slow $(0.25-0.5 \mathrm{~Hz})$, and very fast $(100-500 \mathrm{~Hz})$.

One early application of EEG was in differentiating stages of sleep, with the first comprehensive proposal advanced by Loomis, Harvey, and Hobart (1937). Most recently (Silber et al., 2007), the American Academy of Sleep Medicine has identified five stages: wakefulness, rapid eye movement (REM) sleep, and three stages of lower-frequency, non-REM sleep (NREM 1, 2, and $3)$.

When people wind down at night, the beta and gamma waves of the cognitively active brain typically yield to more relaxed alpha waves, which in turn become mixed with yet slower theta waves as sleep approaches. By definition, an individual is awake (stage $\mathrm{W}$ ) as long as alpha exceeds theta activity, and makes the transition to NREM 1 sleep (stage N1) when theta exceeds alpha activity. For those with little alpha activity, typical accompaniments such as slow rolling eye movements are counted instead. NREM 2 sleep (stage N2) is characterized by one or more half-second (or longer) episodes of high-amplitude patterned activity superimposed on a lowamplitude, mixed-frequency background. One pattern, the sleep spindle, is a train of rhythmic $11-16 \mathrm{~Hz}$ waves that increase and then decrease in amplitude, producing a spindle shape in the EGG. The other pattern, the K-complex, is a sharp negative wave immediately followed by a slower positive component. NREM 3 sleep (stage N3) begins when at least $20 \%$ of activity is slow wave sleep, restricted by definition to the lower end of the delta range $(0.5-2 \mathrm{~Hz}$, with peakto-peak amplitude above $75 \mu \mathrm{V}$ ). Typically these slow waves rise to over $50 \%$ of EEG activity, treated as the threshold to a separate stage in older systems. Finally, REM sleep (stage R) has a complex definition emphasizing three characteristics that tend to co-occur: its namesake rapid eye movements (during which the most memorable dreaming can occur), low muscle tone, and low-amplitude, mixed-frequency EEG activity (usually predominantly theta as in stage N1, but alpha or sawtooth waves may be prominent). After its discovery in 1953, REM sleep was called paradoxical sleep because the sleeper could not move even though the brain and other systems were active. 
The sequence of stages in a prototypical night begins with $\mathrm{W}$, then $\mathrm{N} 1$, then four or five repetitions of N2-N3-N2-R, but variations are common. It should be kept in mind that the stages are rigorously defined in part to assure comparability across research laboratories; in an actual night's sleep the passage from one stage to another often is gradual or ambiguous. The dynamic character of sleep, and its relative isolation from environmental influences, make it a highly relevant context for investigating edogenous activity in the brain.

In summary, EEG research first showed its worth as a means of differentiating overall brain states. In the early decades it was most usefully applied to the discovery and characterization of stages of sleep in terms of endogenous alpha, theta, and delta rhythms. From Berger forward, researchers also recognized that a variety of cognitively active states were marked by faster rhythms in the beta and gamma ranges. They lacked tools for distinguishing between these states, however, as would be needed to move towards a brain-based account of cognitive operations and their orchestration in complex tasks.

This changed beginning in the 1960s (for sensory processing) and 1970s (for more complex cognitive processing). Instead of recording ongoing brain activity-primarily endogenous in origin - investigators presented carefully chosen exogenous stimuli and looked for systematic changes in the EEG pattern, especially within the first half-second or so following stimulus onset. The time-locked waveform is referred to as an evoked potential (EP), evoked response potential (ERP), or in cognitive investigations, event-related potential (also ERP). ${ }^{7}$ This dramatically repositioned the EEG technology: now it could serve those who found a reactive conception of the brain most promising for rapid gains in knowledge. Since response to a stimulus was only one among many influences on the highly variable EEG waveform, however, it was essential to average the waveforms obtained over multiple trials in which similar stimuli were presented. Computer processing of digital EEG made this practical. In a classic experiment, Kutas and Hillyard (1980) presented subjects with 160 seven-word sentences in which the final word was either anomalous or semantically appropriate. Comparing the average ERPs, they

\footnotetext{
${ }^{7}$ There was interest in such waveforms as far back as Berger (1920s) and Davis (1930s); what was new in the 1960s was powerful new tools for identifying and interpreting components.
} 
discovered a robust negative deflection peaking approximately 400 milliseconds after onset of an anomalous word but not after an appropriate word. They interpreted this N400 component as signaling reprocessing of anomalous semantic information and were able to distinguish it from positive deflections signaling disconfirmation of an expectation (the already well-known P300) or following a change in font size.

For our purposes, what is most noteworthy here is the considerable variability across trials in the endogenous components of the EEG waveform that makes it challenging to extract the response specifically evoked by an exogenous stimulus. Although this variability is viewed as noise from a reactive perspective, as mentioned above, it can instead be viewed as reflecting the varied endogenous origins of the brain's ongoing activity. The challenge is to detect and analyze patterns in this activity and uncover their origins and functions. For example, thalamocortical oscillations seem to play a pivotal role in regulating communication between cortical areas (Buzsáki, 2006). We discuss other proposals regarding the functional importance of endogenous activity in section 4 .

\section{b. Recording from Individual Neurons}

EEG rhythms were assumed to reflect neural activity, but what kind of neural activity? Two opposing explanations were pursued in the 1940s and 1950s (see Kaada, 1953). One explanation credited individual cortical neurons with the capacity for endogenous generation of rhythmic firing. If such neurons synchronized their activity, this would be sufficient to produce the overall rhythms observed in an EEG. The other explanation relied on a reactive conception of the neuron now known as integrate-and-fire. It was assumed that each neuron continuously performed an essentially linear integration of inputs received from other neurons at synapses on its dendritic tree. When a threshold was exceeded it "fired"- that is, it sent an electric pulse (called an action potential or spike) along its axon towards yet other neurons. This model was an important part of the conceptual framework for work on the mammalian nervous system, which relied heavily on single-cell recording studies of motor neurons in the spinal cord. The rate of spiking indicated responsivity to a stimulus, and it was relatively straightforward to study propagation through circuits of such neurons. Cortical neurons had much more complex connectivity patterns, but the 
same overall framework was assumed to apply to them. To account for alpha rhythms, for example, it was suggested that a closed, self-re-exciting chain of integrate-and-fire neurons in cortex, driven by thalamic input, could keep neural impulses circulating rhythmically. In the version proposed by Eccles (1951, p. 462) ${ }^{8}$ a $10 \mathrm{~Hz}$ firing pattern—-the alpha rhythm—was assured by the fact that each neuron in the chain required approximately $100 \mathrm{msec}$. of recovery time before it could respond to above-threshold input with an action potential. On this account, endogenous rhythmicity was not required to explain observed rhythmicity.

Support for the alternative conception of the neuron as endogenously active came eventually, from research on invertebrates that was rooted in the dominant reactive perspective but led in new directions. The initial goal was to achieve a finegrained understanding of the action potential itself as an event across time, and the giant axon of the squid provided easy access for intracellular recording under controlled conditions. A key set of researchers were less interested in how fast the electrical pulse traveled down the axon than in the timecourse and mechanism of voltage changes at any point along the axon as the pulse passed. The primary mechanism turned out to be ion movements across the axonal membrane, as captured by Hodgkin and Huxley (1952) in an elegant set of equations. The membrane's resting potential is approximately -65 millivolts, reflecting a normal predominance of negative ions inside and positive ions outside. As a pulse arrives the voltage becomes less negative (depolarizes), triggering an influx of sodium ions $\left(\mathrm{Na}^{+}\right)$from the extracellular space that drives the membrane potential into the positive range (very quickly, due to positive feedback). At a short delay a less rapid efflux of potassium ions $\left(\mathrm{K}^{+}\right)$brings voltage back into the negative range, first overshooting (hyperpolarizing) and then returning to the resting potential.

Thus, the shape of the action potential is derived as the net effect of incoming sodium and outgoing potassium currents. It was not until the 1970s to 1980s that researchers discovered the molecular mechanism behind these dynamics: proteins in the membrane act as specialized ion

\footnotetext{
${ }^{8}$ Eccles bemoaned the confusing accounts of cortical potentials and advocated the study of motor neurons in the spinal cord as affording a clearer perspective on neural activity: "It is the thesis of this paper that basically the responses of neurones are similar throughout the central nervous system, and that the more easily analysed responses of motoneurones provide the data for a satisfactory explanation of the electrical responses evoked in the cerebral cortex by all conditions of stimulation: by direct electrical stimulation; by afferent volleys; and by antidromic volleys" (Eccles, 1951, p. 449).
} 
channels, opening (or closing) as a function of voltage in the immediate vicinity and thereby collectively offering high (or low) conductance to their particular type of ion (see Hille, 2001). Voltage changes at one location on the membrane trigger channel-opening nearby, resulting in propagation of the action potential along the axon.

Although the Hodgkin-Huxley equations were nonlinear, they fit into the dominant reactive framework of the era in that the dynamics they described were those of a neuron firing in response to a depolarizing input. Invertebrate researchers began moving towards an appreciation for endogenous activity in the 1960s, however, as new findings emerged from intracellular recording and related experiments. Notably, investigators found specialized pacemaker neurons that generated their own rhythmic action potentials (Alving, 1968), as well as an unexpected variety of voltage-gated and other currents producing complex dynamics not only in axons but also in neurons' dendrites and cell bodies (reviewed by Kandel, 1976).

Mammalian researchers initially doubted the generality of such findings, but took notice when Rodolfo Llinás and colleagues found a variety of functionally important ion currents in neurons of the inferior olive and cerebellum in mammals (and birds) in the 1970s and 1980s. Most were spatially distributed and gated by voltage differently than the sodium and potassium channels in the axon, equipping them for functions other than the direct generation of action potentials. Notably, the dentrites were endowed with channels providing high-threshold conductance to calcium $\left(\mathrm{Ca}^{2+}\right)$ ions, enabling dynamically complex dendritic excitation in contrast to earlier assumptions of passive transmission of signals from synapses. ${ }^{9}$ Moreover, the cell bodies of some neurons in the inferior olive had a different kind of calcium channel with a seemingly paradoxical low-threshold conductance that, in interaction with sodium and high-threshold calcium conductances, enabled these neurons to function as single-cell oscillators "capable of self-sustained rhythmic firing independent of synaptic input” (Llinás, 1988, p. 1659). ${ }^{10}$ They sent

\footnotetext{
${ }^{9}$ This linked nerve excitability with the $\mathrm{Ca}^{2+}$-dependent second messenger system that is important for regulating general cellular functions. It also provided a mechanistic explanation of the suggestion (Bremer, 1958) that EEG primarily reflects synchronized post-synaptic potentials in dendrites — not, as originally thought, action potentials in axons.

${ }^{10}$ For further exposition, see Buzsáki (2006, pp. 181-183), who comments: "These findings . . . illustrate that nature went to a lot of trouble bringing together these channels at the right densities and location just to serve one purpose: oscillation." For evidence extending the findings to sensory neurons in various mammalian species, see Huguenard (1996).
} 
these rhythmic action potentials to target neurons in the cerebellum that were able to respond at the same frequency, qualifying them as resonators in the dynamical lexicon championed by Llinás_-reacting, but in ways shaped by their internal properties. Llinás also investigated spontaneous oscillations in electrical potentials elsewhere in the brain. Research on the thalamus and thalamocortical relay neurons (e.g., Jahnsen \& Llinás, 1984) proved particularly useful for linking dynamic behavior of individual neurons to the large-scale dynamics seen in EEG.

Llinás' research offers a radically different picture of neural activity than that featured in the reactive framework. Neurons, on his account, are complex dynamic systems that are constantly changing their states and spontaneously generating action potentials. To generate oscillations at the different frequencies found in EEG requires the synchronization of many individual neurons, but before neurons can synchronize they must first oscillate. By showing how they do so, Llinás identified the needed foundation for the overall brain to exhibit complex dynamics. A number of researchers have subsequently built upon this foundation, some examining how multiple ion channels contribute to the intrinsic oscillatory activity of neurons and others determining how different neurotransmitters and receptors affect the temporal dynamics of synaptic activity (see Destexhe \& Sejnowski, 2003, for a review and theoretical framework for modeling how these endogenous and reactive processes interact in producing synchronous thalamocortical oscillations). We return to this in section 5 .

\section{c. Functional magnetic resonance imaging (fMRI)}

Single cell recording has been the workhorse technique of neuroscientists using animal models to understand information processing in the brain, as seen in section 2, but with rare exceptions it is too invasive for studying the human brain itself. It was the advent of functional neuroimaging techniques that catalyzed study of the involvement of different brain areas in a variety of human cognitive performances and gave rise to the distinct field of cognitive neuroscience. An existing technology, positron emission tomography (PET), was adapted to this end in the 1980s by Marcus Raichle and colleagues (as reviewed by Posner \& Raichle, 1994), followed in the 1990s by functional magnetic resonance imaging (fMRI). Each of these techniques detects changes in blood flow in the brain that serve as a proxy for neuronal processing; most commonly used today 
is the BOLD (blood oxygen level dependent) signal from fMRI. Until recently the primary strategy in both PET and fMRI studies has been to identify areas exhibiting higher signal intensity (greater blood flow) when a subject is performing a target task than when performing a control task. Those areas are said to be activated by whatever type of processing distinguishes the two tasks.

If the stimulus is a word, for example, providing a semantically associated word versus merely reading the stimulus word aloud would call upon semantic processing and thereby activate brain areas responsible for the relevant semantic operations (Petersen et al., 1989). Often passive viewing of the same stimulus is included as a control; in the same experiment, reading a word aloud versus passively viewing it identified areas responsible for certain phonological operations. Subtracting brain images to find activated areas has been a powerful strategy for localizing cognitive operations in the brain, currently reaching a spatial resolution as small as $2 \mathrm{~mm}$. It is a clear success story for the reactive paradigm.

There is a twist, though, that has brought the endogenous perspective back into the story. In the decades before neuroimaging was deployed on humans responding to stimuli in cognitive tasks, a few researchers measured blood flow and brain activity in what has been called the resting state. For Berger (and his successors even today) this was the most appropriate state for detecting endogenous activity, especially alpha waves. In contemporary experimental designs, the term references a control condition in which a subject is still with eyes closed and is presented with no stimuli or task requirements (in variations, the eyes are open with or without a fixation point). In a pioneering study using the xenon 133 inhalation technique to measure regional cerebral blood flow, Ingvar (1975) discovered that subjects at rest exhibited high levels of frontal activity. He surmised that this activity reflected "undirected, spontaneous, conscious mentation, the "brain work,' which we carry out when left alone undisturbed" (quoted by Buckner et al., 2008, p. 2). In an even earlier study using nitrous oxide to measure cerebral metabolism, Sokoloff, Mangold, Wechsler, Kennedy and Kety (1955) found that performing mental arithmetic did not increase metabolism, indicating that the background activity was as energy demanding as any operations involved in performing a cognitive task. Recently, Raichle and Mintun (2006) drew upon these and other findings to argue that while the brain consumes $20 \%$ of the energy utilized in the body, 
it increases its consumption very little when performing tasks rather than resting. Humans exhibit, it is then inferred, a great deal of endogenous activity even in the resting state.

The early results indicating substantial endogenous activity received little further attention once PET and fMRI burst on the scene and researchers focused on identifying brain areas activated in experimenter-defined tasks. Often, though, they included the resting state as a control condition, presuming that it would elicit less activation than any of the tasks. A few of these researchers were intrigued to find that certain brain areas around the midline instead exhibited greater activation (stronger BOLD signal) in the resting state condition than in task conditions (e.g., Ghatan et al., 1995; Baker et al., 1996). They characterized these areas as deactivated during tasks, but it should be kept in mind that this referred to low relative activity (not negative activity). The choice of term reflects surprise that some of the subtractions (task condition minus rest condition) would yield negative numbers.

To determine whether a common set of brain areas manifested task-induced deactivation, Shulman et al. (1997; see also Mazoyer et al., 2001) conducted a meta-analysis of PET studies in which a task condition was compared to a non-task condition in which the same stimulus was present (which turned out to be similar in result to a resting condition with no stimulus). They found that the following areas were reliably less active in task situations, as shown in Figure 5: the junction of precuneus and posterior cingulate cortex (PCC), inferior parietal cortex (IPC), left dorsolateral prefrontal cortex (left DLPFC), a medial frontal strip that continued through the inferior anterior cingulate cortex (inferior ACC), left inferior frontal cortex, left inferior frontal gyrus, and the amygdala. 


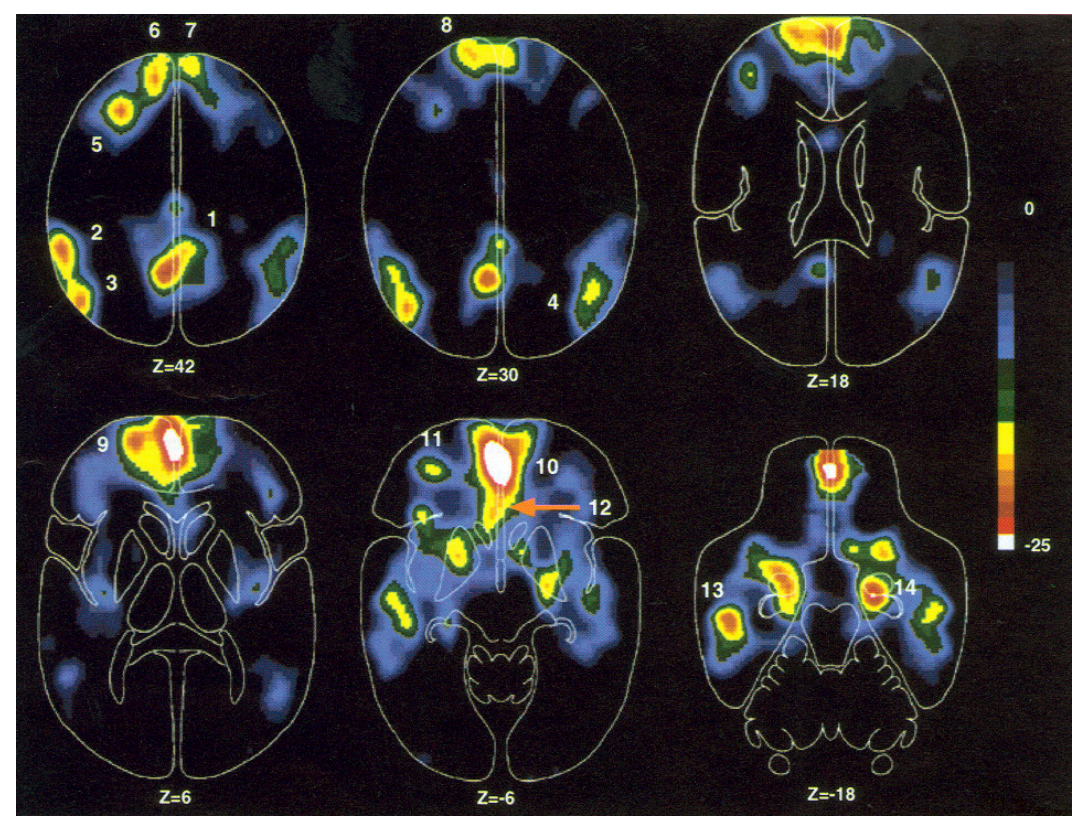

Figure 5. Metaimage from Shulman et al. (1997) in which areas showing decreases in blood flow during task performance versus resting state are indicated in yellow and red. Components of this default network are labeled by numerals as follows: Junction of posterior cingulate and precuneus (1); Inferior parietal cortex $(2,3,4)$; Left dorsolateral prefrontal cortex (5); Medial frontal strip that continues through the inferior anterior cingulate cortex $(6,7,8,9,10,12)$; Left inferior frontal cortex (11); Left inferior temporal gyrus (13); Right amygdala (14).

Shifting the focus from the fact that these areas are less active during tasks to the fact that they are more active in the absence of task requirements, Raichle and his collaborators (Raichle et al., 2001; Gusnard et al., 2001) proposed that these areas constitute a default network - one which performs actual functions best carried out when there are no external task demands. There are clues to those functions in Ingvar's 1975 study, discussed above, and more directly in a neuroimaging study of autobiographical memory. Andreasen et al. (1995) found that the areas exhibiting heightened BOLD responses in a resting state condition were also relatively active in an episodic memory task. In contrast, a different set of areas exhibited heightened BOLD responses in a more typical semantic memory task. In an attempt to figure out what functions might elicit increased activity during rest, the researchers queried the subjects. Their reports pointed towards "a mixture of freely wandering past recollection, future plans, and other personal thoughts and experiences"-activities that plausibly draw upon episodic memory. Subsequent research has confirmed that thinking about one's own experiences is among the characteristic functions of the default network. 
The studies discussed so far focused on relative amount of activity in the default network under various conditions, but not on the micro-temporal dynamics of this activity. Synchronized oscillations would be a salient criterion for network status, but finding them with fMRI initially seemed challenging due to the sluggish nature of the hemodynamic response. The feasibility of such a temporal analysis of fMRI data was demonstrated first for networks activated by tasks. Biswal, Yetkin, Haughton, and Hyde (1995) obtained BOLD signal values every 250 msec. for two minutes following a simple motor task (moving a hand). They identified spontaneous very low-frequency oscillations bilaterally in sensorimotor cortex, i.e., less than one cycle every 10 seconds $(<0.1 \mathrm{~Hz})$. These oscillations were synchronized across the left and right hemispheres and also with oscillations in other motor areas. The researchers interpreted their results as indicting functional connectivity among the regions studied. Cordes et al. (2000) found similar oscillations in resting state BOLD signals in networks of areas previously identified by their synchronized activity in sensorimotor, visual, receptive language, or expressive language tasks. Moreover, their functional connectivity MRI ( $\left.f_{c} M R I\right)$ analysis - applying correlational statistics to resting state BOLD time series data to determine patterns of synchronization - yielded functional networks very similar to those identified from activity during tasks. That is, areas within the same network had correlated patterns of activity across time (rising and falling in synchrony) regardless of whether overall level of activity was relatively high (e.g., the sensorimotor network while moving a hand) or relatively low (e.g., the same network in a resting state condition).

To begin assessing whether the regions proposed to constitute a default network likewise met the criterion of synchronized oscillation, Greicius, Krasnow, Reiss, and Menon (2003) employed fcMRI with two seed areas, the PCC and inferior ACC. They regarded their results as providing "the most compelling evidence to date for the existence of a cohesive, tonically active, default mode network" (p. 256) and argued that the PCC was a critical node in this network. When it was used as the seed area for statistical analysis, its resting state oscillations were correlated with those in much of medial prefrontal cortex (including inferior ACC and orbitofrontal cortex), left DLPFC, IPC bilaterally, left inferolateral temporal cortex, and left parahippocampal gyrus. (One of these synchronies is illustrated, using data from another study, in Figure 6.) This is almost the same set of areas as those deactivated in task conditions according to Shulman's et al.'s meta- 
analysis. Greicius et al. argued that this default network performs higher cognitive functions, especially various forms of endogenously directed memory retrieval. Turning to their findings for ventral ACC as the seed area, the correlated areas included the PCC, medial prefrontal cortex/orbitofrontal cortex, the nucleus accumbens, and the hypothalamus/midbrain. They argued that these primarily paralimbic and subcortical areas comprised a network important for calibrating affective and autonomic operations, and further suggested that the strong connection between inferior ACC and PCC provided a crucial link between the two networks.

Finally, the investigators confirmed that both networks became deactivated during a working memory task but not in an eyes-closed or eyes-open resting state or even when passively viewing a blinking checkerboard. Besides indicating a rather high threshold of cognitive demand for deactivation of the default network, these findings clearly distinguished the default network from the neural system responsible for alpha rhythms in EEG (which diminish when subjects open their eyes). Subsequently Greicius and Menon (2004) found that the default network included the hippocampus and Vincent et al. (2006) determined that by seeding an analysis with a hippocampal region they could find correlated activity in the rest of the default network. ${ }^{11}$ Buckner, Andrews-Hanna, and Schacter (2008, pp. 4-5) summed up the perspective provided by this research: "The default network is a brain system much like the motor system or the visual system. It contains a set of interacting brain areas that are tightly functionally connected and distinct from other systems within the brain."
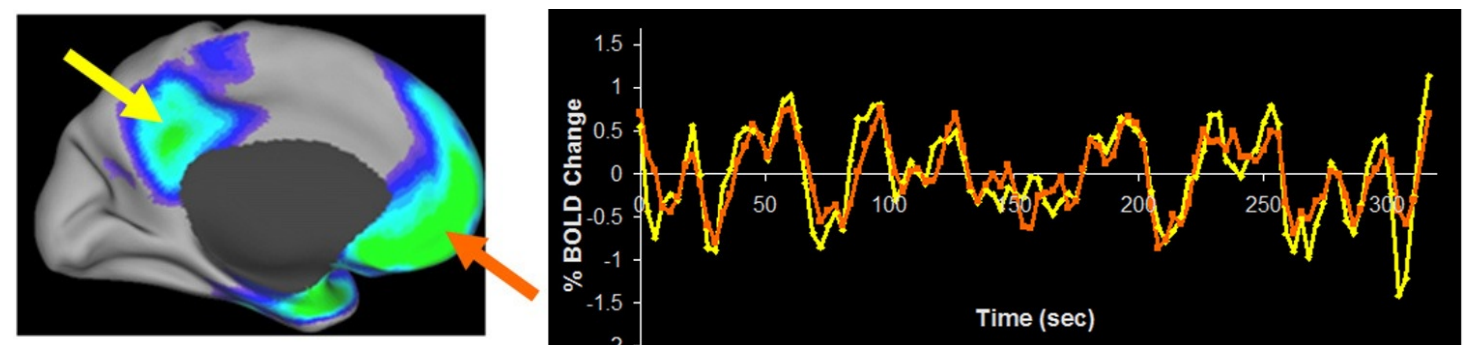

Figure 6: On the left, two areas of the default network are indicated: Posterior cingulate cortex (yellow arrow) and ventral medial prefrontal cortex (orange arrow) On the right, it can be seen that patterns of activity for the two areas are highly correlated. From Raichle and Synder (2007).

\footnotetext{
${ }^{11}$ By combining time-series analysis of fMRI with diffusion tensor imaging, van den Heuvel, Mandl, Kahn, and Pol (2009) have recently shown that eight of nine areas in the default network are directly connected by fiber tracts (see also Greicius et al., 2009).
} 
Most of the known networks in the brain, in contrast to the default network, show more BOLD activation during tasks than at rest. But even at rest there is enough activity to assess whether the constituent areas of any such task-activated network fluctuate in synchrony with each other (but not with the default network). Fox et al. (2005) selected a network that was especially active during attention-demanding tasks (intraparietal sulcus, frontal eye field, middle temporal region, supplementary motor areas, and the insula). Examining those areas in the resting state, they found that fluctuations in their BOLD signals indeed were correlated. Moreover, fluctuations in that network and in the default network were anticorrelated. That is, the areas that were positively correlated within each network were negatively correlated with areas in the other network - an outcome more interesting than a zero correlation. Fox et al. emphasized that: anticorrelations may be as important as correlations in brain organization. Little has been said previously in the neuronal synchrony literature regarding the role of anticorrelations. While correlations may serve an integrative role in combining neuronal activity subserving similar goals or representations, anticorrelations may serve a differentiating role segregating neuronal processes subserving opposite goals or competing representations (p. 9677).

This pattern of results in resting state data is a strong indicator that within both the default network and the network involved in attention-demanding tasks, coordinated activity of some kind goes on in the absence of external stimulation - activity that is is different for the two networks.

Subsequently, researchers have used the strategy of finding correlations in resting-state fluctuations to identify yet other networks. For example, temporally correlated activity was found by Vincent et al. (2006) in the hippocampus and parietal memory systems and by Fox, Corbetta, Synder, Vincent, and Raichle (2006) in the dorsal and ventral attention systems. Deploying an alternative method, independent component analysis, on resting state fMRI data, Mantini, Perrucci, Del Gratta, Romani and Corbetta (2007) differentiated six different networks. ${ }^{12}$ Fox and Raichle (2007, p. 701) concluded: “A consistent finding is that regions with similar functionality — that is, regions that are similarly modulated by various task paradigms-

\footnotetext{
${ }^{12}$ An interesting finding in their study was the differentiation of the default network from what they characterize as a "self-referential network" that contains areas often associated with the default network: medial-ventral prefrontal cortex, the pregenual anterior cingulate, the hypothalamus, and the cerebellum.
} 
tend to be correlated in their spontaneous BOLD activity." "13 An important unanswered question is how the anticorrelation is achieved — via a separate control system or as a direct result of competition between networks.

\section{d. Relating endogenous oscillations at different timescales}

Spontaneous oscillations in brain activity for awake humans are found across a wide range of timescales, depending on measurement technology and practices. Characteristic oscillations are slowest in fMRI data $(<0.1 \mathrm{~Hz})$, midrange in EEG $(1-80 \mathrm{~Hz})$, and fastest in single-cell recording of action potentials $(>100 \mathrm{~Hz})$. This raises the question of whether neural activities as captured by different methods at different timescales are independent or related.

Intriguing results have been reported by researchers who asked whether the networks inferred from correlated BOLD signals in fMRI exhibit distinctive EEG signatures. Laufs et al. (2003) first found that activation of the default network is associated with strong activity in the mid-beta range, and activation of attention-related frontal and parietal areas is associated with weak activity in the alpha range, i.e., alpha blocking. Mantini, et al. (2007) expanded this kind of analysis to the six networks they had identified from resting state fMRI data, seeking associations across as a broader range of EEG frequency bands. They found that "Each brain network was associated with a specific combination of EEG rhythms, a neurophysiological signature that constitutes a baseline for evaluating changes in oscillatory signals during active behavior" (p. 13170). Their methods were sensitive enough to find alpha and beta rhythms together providing a broader positive signature for the default network and negative signature for the attention network. Beta recombined with delta and theta rhythms as the positive signature for the auditory network and was the sole positive signature for the somatomotor network. Gamma rhythms were the only positive signature for the self-referential network and the only frequency band excluded for the vision network. It was assumed that different sets of neurons within an

\footnotetext{
${ }^{13}$ Fox and Raichle also note that finding correlations in the resting state ("spontaneous BOLD activity") is a good way of identifying the full range of components of a network, since many of them may not be activated by any particular task. "Interestingly, most memory tasks implicate only a subset of regions, whereas the hippocampal formation resting-state correlation map reveals the full distribution of memory-related regions assessed across multiple experiments. Patterns of spontaneous activity could thus serve as a functional localizer, providing a priori hypotheses about the way in which the brain will respond across a wide variety of task conditions" (p. 702).
} 
area were responsible for different rhythms, but also that the resting state BOLD signal indirectly reflected the endogenous activity of those same neurons.

In another line of research, direct-current-coupled full-band electroencephalography has been used to detect infraslow oscillations (.01 - .1 Hz, meaning a single cycle lasts 10 to 100 seconds). The rising phase of a single ultraslow cycle is associated with (a) higher-amplitude activity in each of the traditional EEG frequency bands and (b) improved accuracy at detecting a somatosensory signal (Monto et al., 2008). Infraslow EEG oscillations are similar in timescale to BOLD oscillations in $\mathrm{FMRI}$, and there are indications that these are sensitive to some of the same activity. For example, He, Snyder, Zempel, Smythe, and Raichle (2008) found a positive correlation between slow cortical potentials (infraslow and delta activity combined) and spontaneous fluctuations in the BOLD signal.

A final example of interrelatedness can be found in analyses of local field potentials, which are recorded in animals by means of electrodes implanted in the extracellular space between neurons. A variety of neural activities contribute to this signal. If dendritic and other lowerfrequency activities are of primary interest, high frequency oscillations reflecting action potentials $(>150 \mathrm{~Hz})$ can be removed. The highest-amplitude oscillations contributing to the remaining signal in monkeys turn out to be in the same frequency range $(<0.1 \mathrm{~Hz})$ as the oscillations in the human BOLD signal (Leopold et al., 2003).

\section{The Significance of Endogenous Oscillatory Brain Activity}

In the previous section we reviewed evidence from EEG, single-cell recording, and fMRI studies pointing to endogenous oscillatory activity in the brain. An advocate of the reactive framework might acknowledge these findings but downplay their significance, maintaining that these oscillations are appropriately regarded as noise with respect to any functional analysis of neural performance. For example, one would expect basic metabolic activities to be maintained even in the absence of task demands, so perhaps the endogenous electrical activity is merely an epiphenomenon of ongoing metabolism. However, the evidence we reviewed indicated that the oscillations within individual brain areas are periodic, not random, and that those areas are 
organized into networks within which oscillations are correlated and between which they are anticorrelated. As well, these networks exhibit distinctive EEG signatures. This intricate organization is highly persuasive that the endogenous activity in the brain is functional, and invites the thought that this functional activity is so important that it cannot be ignored in seeking to understand how the brain performs its functions. Here we briefly explore four different ways in which endogenous activity may be crucial for understanding the brain mechanisms involved in cognition.

First, it seems obvious that for any mechanism that responds to stimuli with an increase (or decrease) in activity, but also exhibits ongoing endogenous oscillations, the magnitude of any particular response will depend in part on the phase of that oscillation at the moment the stimulus arrives. Fox, Snyder, Zacks, and Raichle (2006) devised an innovative strategy for demonstrating that this is true of neural activity in somatomotor cortex (SMC). Specifically, they showed that trial-to-trial variability in fMRI BOLD response to an exogenous stimulus could be attributed largely to spontaneous (endogenous) fluctuations. Data were available from subjects who had been instructed to press a button with the right hand each time an event was detected. This evoked a BOLD response in left SMC, peaking on average at eight seconds following the button press and then returning to baseline. However, there was considerable variability across trials. Typically such variability is treated as random noise, but Fox et al. suspected that it reflected endogenous oscillations on which the event-related responses were superimposed. That is, the endogenous BOLD signal might happen to be relatively high or low or inbetween at the moment a particular button press was required. The researchers surmised that simultaneous spontaneous fluctuations in right SMC should serve as a good proxy for the endogenous contribution to the activity in left SMC, since these were correlated in resting state and only left SMC was involved in production of a right-hand button press. Indeed, they found that the task-related increase in the left SMC BOLD signal could be analyzed as a linear addition to the amplitude of the spontaneous fluctuation. A subsequent study (Fox et al., 2007) was designed to permit calculations of trial-to-trial variability in the BOLD amplitude at each of eight timepoints following a button press. As illustrated in Figure 7, variability in left SMC was significantly reduced by subtracting out the corresponding activity in right SMC. This indicated that much of the variability reflected ongoing spontaneous fluctuations - not noise - and that event-related 
activity is superimposed on these fluctuations. ${ }^{14}$ Moreover, they were able to extend previous findings of an association between behavioral and BOLD responses by showing that the force with which the button was pressed was correlated with the spontaneous fluctuation component in their own BOLD data. There were some complications in this BOLD-behavior effect that await further research for firm interpretation, but the main message is clear: spontaneous fluctuations in brain activity are synchronized within networks and contribute to the variability of eventrelated responses in both brain and behavior.
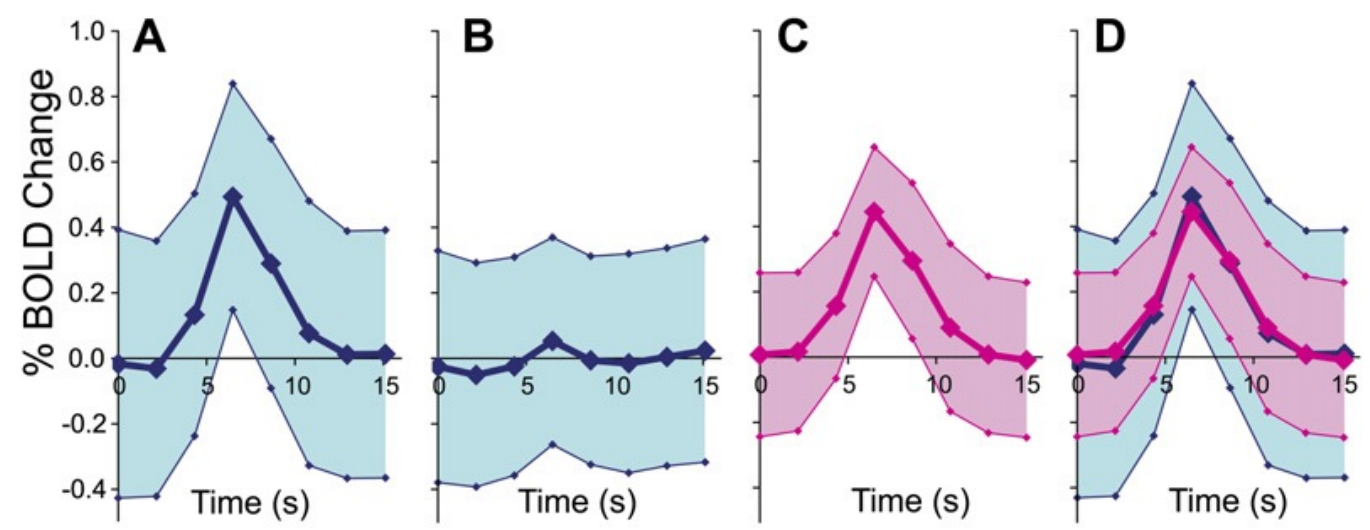

Figure 7. Fox et al.'s (2007) demonstration that (A) the variability (average standard deviation) around the mean BOLD response in left SMC in a button press task is largely explained by (B) the spontaneous activity in the right SMC. Panel (C) shows the effect of subtracting (B) from (A).

Panel D is an overlay of panels (A) and (C) showing in blue the variability attributable to spontanous activity and in light blue the remaining unexplained variability in BOLD activity in the left SMC.

Second, at the end of the previous section we noted that oscillations recorded through EEG are positively correlated with the slower BOLD oscillations registered in fMRI. These correlations may reflect systemic coherence in brain functioning. It has been found that when a mammalian EEG waveform is decomposed into simpler component waveforms (e.g., by fast Fourier transform), the amplitude of each component is inversely proportional to the frequency (1/f). (See Freeman et al., 2000.) Even more interesting, there is evidence that the amplitude of higherfrequency oscillations is modulated by the phase of lower-frequency oscillations. Specifically, gamma waves $(30-50 \mathrm{~Hz})$ are strongest during the rising-positive portion of a single theta wave

\footnotetext{
${ }^{14}$ There had been similar findings earlier at the level of individual neurons. In a single cell recording study using cats, Tsodyks, Kenet, Grinvald, and Arieli (1999) showed that a given neuron's response to a visual stimulus was affected by the spontaneous state of the visual system at the time of stimulation, as indicated by local field potentials measured by means of optical imaging with voltage sensitive dye.
} 
(4-10 Hz) within which they are embedded, and in turn the small number of theta waves concomitant with a single delta wave (1-4 Hz) are strongest during its rising-positive phase. (These data and the "oscillatory hierarchy hypothesis" proposed to explain them are from Lakatos et al., 2005; see also Canolty et al., 2006.) Such coupling can be particularly important when the brain is perturbed by a stimulus, since a modulation in low-frequency oscillations can, through phase-locking with higher-frequency oscillations, yield rapid changes at those frequencies. In addition, oscillations at lower frequencies tend to synchronize over more widely distributed areas of the brain than those at higher frequencies:

[The] $1 / f$ power relationship implies that perturbations occurring at slow frequencies can cause a cascade of energy dissipation at higher frequencies and that widespread slow oscillations modulate faster local events. These properties of neuronal oscillators are the result of the physical architecture of neuronal networks and the limited speed of neuronal communication due to axon conduction and synaptic delays. Because most neuronal connections are local, the period of oscillation is constrained by the size of the neuronal pool engaged in a given cycle. Higher frequency oscillations are confined to a small neuronal space, whereas very large networks are recruited during slow oscillations" (Buzsáki \& Draguhn, 2004, p. 1926)

In sum, as a result of endogenous activity maintaining synchronized oscillations at different frequencies, the brain is able to generate coordinated responses to perturbations.

Third, endogenous activity in the brain's default network is the most obvious candidate for the neural underpinnings of mindwandering (Antrobus et al., 1970). Ingvar's (1975) interpretation of his early blood flow studies noted above suggested such a connection. Further support was provided by Andreasen et al.'s (1995) subjects, whose informal reports after they had been imaged in the resting state emphasized recollection and planning. These activities involve episodic memory, and episodic memory tasks are among the few highly cognitive tasks for which the default network's BOLD signal remains as strong as in the resting state. Buckner and Carroll (2007) in fact found that remembering the past, envisioning future events, and considering the thoughts and perspectives of other people produced selective activation within the default network. They construed these results as supporting the view that activity recorded during the resting state reflects thinking — but thinking that is "engaged when individuals are left 
to think to themselves undisturbed" rather than elicited by specific stimuli or tasks (Buckner et al., 2008, p. 1). They then hypothesized "that the fundamental function of the default network is to facilitate flexible self-relevant mental explorations - simulations - that provide a means to anticipate and evaluate upcoming events before they happen" (p. 2). ${ }^{15}$ In defending this view they cite not only Andreasen at al.'s results but also correlations found by Mason et al. (2007) between stimulus independent thoughts (as initially characterized by Antrobus et al., 1970) and activity in the default network. Intriguingly, Li, Yan, Bergquist, and Sinha (2007) correlated trials on which subjects failed to detect stop signals in behavioral tasks with increased activity in the default network, as one would expect if that network were involved in a person thinking distracting thoughts about past and future experiences.

One factor that renders problematic such a characterization of the activity of the default network is that its oscillatory behavior is well maintained in sleep (Fukunaga et al., 2006; Larson-Prior et al., 2009) and under anesthesia (Vincent et al., 2007) — circumstances in which spontaneous conscious thoughts (such as Andreasen et al.'s subjects report) presumably are not occurring. This suggests that the default network's spontaneous activity is more foundational than originally supposed. Fox and Raichle (2007) considered three possible interpretations of synchronization in such activity between different areas in this network, as evidenced in correlated BOLD signals; these interpretations have in common that they reflect cognitive processing but need not be characterized in terms of conscious thought:

One possibility is that spontaneous activity serves as a record or memory of previous use, showing correlations between regions that have been modulated together in a taskdependent manner. Another possibility is that spontaneous activity serves to organize and coordinate neuronal activity and that this coordination is more prominent between regions that commonly work in concert. This is similar to the temporal binding, although spontaneous BOLD occurs at a much slower, broader and more permanent scale. Finally, spontaneous activity may represent a dynamic prediction about expected use, with

\footnotetext{
${ }^{15}$ They also presented, but did not pursue, an alternative view that activity in the default network generates lowlevel generalized awareness or watchfulness (Gilbert et al., 2007). This view gains support from Hahn, Ross, and Stein's (2007) findings of increased activity in the default network in a target-detecting task when the target could appear anywhere, but not when it was expected in a specific location.
} 
correlations occurring between regions that are likely to be used together in the future ( $\mathrm{p}$. 709).

Fourth, endogenous brain activity might be crucial for building and maintaining certain types of organization in the nervous system required for cognitive activity. There is growing evidence that the brain exhibits small-world organization (Watts \& Strogratz, 1998) in which most connections are between neighboring neurons, creating clusters that can collaborate in processing specific information, but a few long range connections enable overall coordination. There also is evidence that while most brain areas have connections to only a few other areas, some have a large number of connections, thereby constituting hubs. Both neuroanotomical and neurophysiological studies provide compelling evidence of such an architecture at different scales in the brains of different organisms. Watts and Strogatz, for example, identified a smallworld architecture in the neural network of the nematode worm Caenorhabditis elegans, whose structure had been identified by White, Southgate, Thomson, and Brenner (1986) using serial reconstruction of electron microscopy sections. Also, as shown in Figure 8, Sporns and Zwi (2004) developed a connection matrix for the 30 cortical areas and 311 connections Felleman and van Essen (1991) identified in the macaque visual cortex and demonstrated that it exhibited the features of a small-world network: short characteristic path lengths and high clustering. They determined that some areas, such as V4, exhibit an atypically large number of connections to other areas, qualifying them as hubs. 


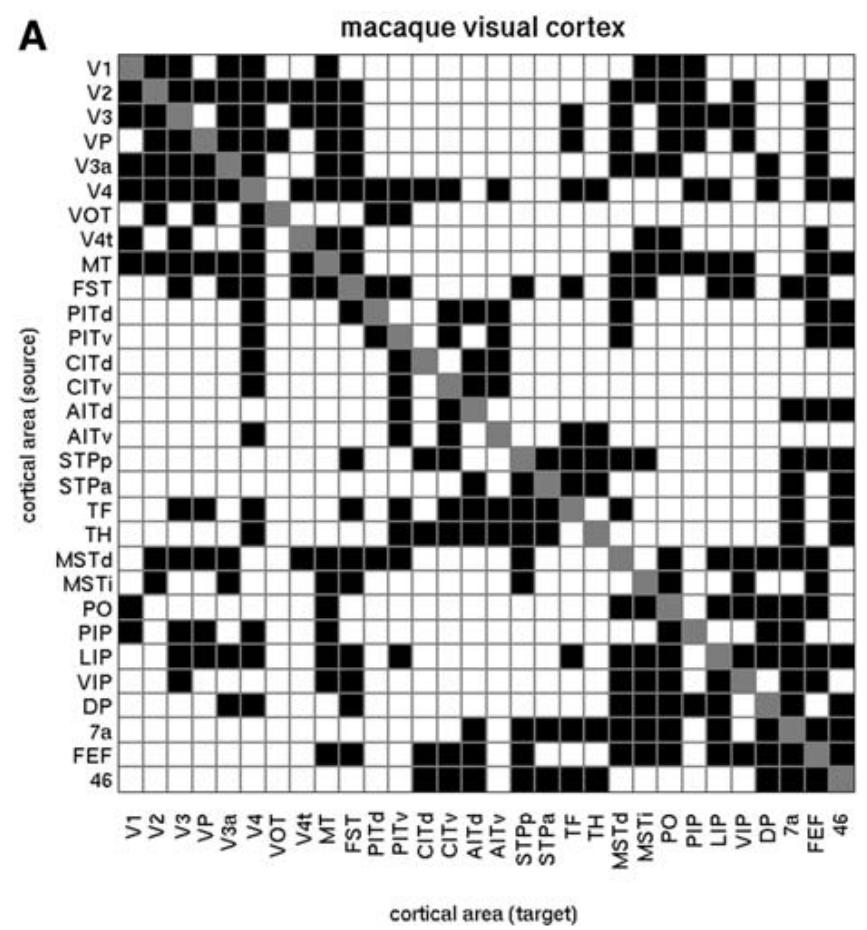

Figure 8. Sporns and Zwi's (2004) connection matrix based on Felleman and van Essen's (1991) depiction of connectivity in the macaque visual cortex (shown in Figure 3). Black squares indicate a known connection and white squares indicate that no connection has been identified. The connections are neither random nor purely local, but clustered so as to indicate a small-world network architecture.

Such an architecture provides a highly efficient organization for information processing. It is notable that the default network itself exhibits a small-world architecture, as indicated by neuroanatomical studies on macaque homologues to the areas composing the human default network. Some of these areas (PCC/Rsp, vMPFC, and IPL) are hubs that link the other areas into a network. An important question is how such organization might arise. Rubinov, Sporns, van Leeuwen, and Breakspear (2009) advanced the intriguing suggestion that oscillatory neurons, by developing connections when synchronized, might self-organize into a small world network with hubs. In support of this proposal they described a model by Gong and van Leeuwen (2004) that employed a logistic map activation function for individual units that can individually exhibit chaotic dynamics. Including a coupling factor enables the emergence of temporary patterns of synchronized oscillations. A Hebbian learning procedure establishes new connections between pairs of units whose activity is synchronized and prunes those between unsynchronized units. 
Even when these networks begin with random connectivity, they develop clusters linked to each other through hubs.

Once this structure appears, it in turn generates more coherent patterns of synchronized activity in subsequent processing, which leads via further Hebbian learning to a structural organization that is likely to exhibit yet more coherent synchronized patterns. At the larger scale of human brains, symbiotic interaction between the generation of functional synchrony and the building of structural connectivity could well result in a highly coordinated brain capable of maintaining multiple anticorrelated networks. The initial state in real brains presumably is already biased towards a small-world pattern of connectivity, rather than random connections as in the simulations, and experience further shapes the emerging organization by Hebbian or other kinds

of learning. Even so, the endogenous activity may be an important molding force so that the very architecture of the information processing system is in an important way a consequence of both endogenous and evoked activity.

In this section we have considered four suggestions as to how endogenous activity in the brain may contribute to its functioning as a cognitive system. Although it is too early to judge which construals will prove most fruitful, clearly the time for dismissing endogenous activity as mere noise has passed.

\section{Conclusion: Endogenous Brain Activity and Dynamic Mechanistic Explanation}

In the last two sections we provided an overview of the now substantial body of evidence that the brain is an endogenously active mechanism (or assemblage of mechanisms), one that is perturbed by stimuli or task impositions but changes its activity in ways that depend not just on these "inputs" but also on internal dynamics. This contrasts with the reactive framework of the vision research discussed in section 2 , in which brain activity is treated as a response evoked by a stimulus. Llinás suggested viewing the interaction between stimuli and the brain's endogenous activity as a conversation:

Although sensory nerve pathways deliver messages to the CNS that are quite invariant with respect to given sensory stimuli, the manner in which the CNS treats these messages 
depends on the functional state of each relay station. Thus, rather than a simple mirror of the external world, the CNS embodies a dialogue between the internal states generated by the intrinsic electrical activity of the nerve cells and their connectivity, which represents the internal context, and the information that reaches the brain from the senses (Llinás, 1988, p. 1633).

As with spoken dialogue, we are unlikely to understand how the brain subserves cognitive activity if we listen to only one side of the conversation: the message conveyed by a stimulus. It is equally important to identify endogenous activity and its contribution to what the system does next.

In this concluding section we return to the question of what conception of mechanism is best suited to understanding the brain's endogenous activity. Neuroscience has a long history of offering explanations in terms of basic mechanisms: parts and operations organized to produce a phenomenon, with that organization described qualitatively rather than quantitatively. The definition offered by Machamer, Darden, and Craver (2000) adds the stipulation that the mechanism's activity leads from set-up to termination conditions, which suggests sequential organization and situates basic mechanistic explanation within the reactive perspective that has long dominated research. This approach has been highly productive, but it offers insufficient resources for understanding the dynamics of endogenous activity. Oscillations and other complex dynamics arise only in mechanisms with nonlinear component operations exhibiting some sort of nonsequential or cyclic organization (particularly feedback loops). Thus, a conception of mechanism adequate for endogenously active systems must reveal how nonsequentially organized, nonlinear operations interact quantitatively to generate the overall behavior of the mechanism. But when we as humans try to understand a particular system's behavior, we find it difficult to go beyond mental rehearsal of a sequence of operations; that is, our cognitive limits predispose us to basic mechanistic explanation. To understand endogenously active mechanisms we must turn to strategies that extend our capabilities.

The main scientific strategy for understanding how mechanisms generate complex behavior is computational modeling, in which researchers mathematically describe the dependency of changes in certain variables on changes in other variables, often by means of differential 
equations. As discussed in section 1, some computational models are explicitly grounded in mechanistic accounts; that is, particular variables or other terms in their equations correspond to particular properties of the mechanism's parts and operations. Given such an alignment of variables with properties, the modeler can simulate the functioning of the mechanism by assigning initial values to variables and performing numerical integration with an appropriate time step. The time evolution of each variable (the timecourse of its values) will depend in part on these initial values, but also on the constants chosen for the model's parameters. Sometimes biologically plausible values that have worked well in other simulations are known; if not, the modeler can determine best-fit values or, more interesting, determine how different values affect the model's behavior. Not infrequently, computational models can be best understood by employing the concepts and representational tools of complexity or dynamical systems theory, with possible implications for the mechanistic account. For example, when the time evolution of two of the model's variables meet criteria for a limit cycle in phase space, this indicates that the mechanism is capable of producing sustained oscillations.

Thus, the type of explanation required is one that integrates these strategies: dynamic mechanistic explanation. Mechanistic explanation identifies the parts and operations of a mechanism and how they are organized, and computational modeling and tools of dynamical systems theory reveal how such a mechanism will behave. This distinctive type of explanation is playing an increasingly important role in the sciences and warrants attention in the new mechanistic philosophy of science as well (Bechtel \& Abrahamsen, 2010, 2011).

An instructive example of dynamic mechanistic explanation is provided by accounts of the endogenous oscillatory capabilities of thalamocortical (TC) relay neurons. These neurons produce sustained pacemaker oscillations - slow but regular firing at a frequency of 1-3 Hzduring human sleep. The mechanism has been uncovered by in vitro investigations using cat and rat TC neurons (Leresche et al., 1991). These oscillations rely upon the coordination of two voltage-gated currents: (1) $I_{\mathrm{h}}$ is an influx of sodium and potassium ions through channels opened when the membrane gets hyperpolarized following a spike. (2) $I_{\mathrm{T}}$ is an influx of calcium ions brought about when the depolarization due to $I_{\mathrm{h}}$ opens low-threshold calcium channels. The 
ensuing calcium spike ${ }^{16}$ further depolarizes the membrane, causing the various ion channels to close. This results in temporary hyperpolarization of the membrane, and the cycle repeats.

The basic mechanistic account provides a spatial layout of the relevant parts (membrane, ion channels, and ions) and specifies the sequence of their operations. The overall activity of the TC neuron - its firing pattern - will depend on quantitative properties of these parts and operations. Several different computational models aligned with the basic mechanistic account have succeeded in simulating the pacemaker firing pattern. Dextexhe, Bal, McCormick and Sejnowski (1996), for example, used Hodgkin-Huxley style equations that included variables for the conductance and activation of each type of channel, membrane voltage, and other properties of the TC neuron's component parts and operations. ${ }^{17}$

One of the parameters specified the maximum conductance of the $I_{\mathrm{h}}$ current (essentially, the membrane's capacity to move sodium and potassium ions if the maximum number of relevant channels were open). There were values of this parameter at which they succeeded in simulating the pacemaker oscillation pattern. But even more interesting was the fact that values in a higher range changed the dynamics of the conductance of the $I_{\mathrm{h}}$ current relative to that of the $I_{\mathrm{T}}$ current in such a way that the system now exhibited spindle-like oscillations. A series of spikes at about 1-3 Hz with a more or less spindle-shaped amplitude envelope would be observed for a few seconds, followed by a quiet period of five seconds or longer, followed by another series of spikes, then a quiet period, and so forth. This is the pattern that surprised Llinás in the 1980s and drew him and others to a more dynamic conception of the neuron. Finally, there was yet a higher range of values for the parameter that produced a quiet resting state.

\footnotetext{
${ }^{16}$ This refers to a rapid depolarization of the membrane due to an influx of calcium, in contrast to the even more rapid depolarization that characterizes action potentials and involves channels specialized to sodium ions. In both cases the membrane then repolarizes less rapidly as the channels close. Hence, a plot of membrane voltage shows a rapid rise followed by a somewhat less rapid decline, typically overshooting — hyperpolarization — before returning to baseline.

${ }^{17}$ Neither this basic mechanistic account nor the computational model enumerates individual ions and ion channels - there are simply too many. Thus, the basic mechanistic account states that there is large number of lowthreshold calcium channels that can open or close and can vary in permeability. The computational account includes a conductivity variable that is specific to the type of channel and is presumed to represent the collective effect of the number of channels and their properties. Accounts at the level of individual channels are also available, but they focus on how a single channel works.
} 
Destexhe and Sejnowski (2003) provide a comprehensive guide to the many innovative models and research investigations of thalamocortical neurons including subsequent empirical evidence that their different patterns of activity are regulated in the manner proposed in their 1996 model. Buzsáki (2006) makes the case that complex dynamics in various brain regions - not only endogenous oscillations but also synchronized activity — are crucial to understanding how the brain works.

One point to emphasize in closing is that adding a focus on dynamics is not intended to replace the importance of traditional mechanistic research directed at identifying the parts, operations, and overall organization of a mechanism. There are some who disagree, arguing for dynamical explanation as a self-contained, successful competitor to mechanistic explanation (e.g., Chemero $\&$ Silberstein, 2008). Dynamical accounts that are not grounded in accounts obtained by decomposing mechanisms into their parts and operations do describe the activity of possible systems, but those systems may not be be like those that are actually functioning in the world. For a dynamical account to offer explanation, it must characterize activity of the actual mechanism producing the phenomenon of interest. When the variables and terms in a computational model are grounded in properties of well-established parts and operations, we have a better basis for trusting the explanation. On the other hand, the model may reveal that the mechanism, as delineated so far, accounts for some but not all aspects of the actual behavior. Tinkering with the model to determine what sort of mechanism would account for these additional aspects can then serve as a discovery heuristic by predicting the occurrence of new parts, operations, or organizational relationships. Some of these predictions may be borne out, some not, generating a dialectical engagement between mechanistic research and computational model building. Such dialectical engagement may provide the only way neuroscience can account for the endogenous dynamics exhibited in brains and ultimately the cognitive behavior the brain supports. 


\section{References}

Adrian, E. D., \& Matthews, B. H. C. (1934). The Berger rhythm: Potential changes from the occipita lobes in man. Brain, 57, 355-385.

Alving, B. O. (1968). Spontaneous activity in isolated somata of Aplysia pacemaker neurons. Journal of General Physiology, 51, 29-45.

Andreasen, N. C., O'Leary, D. S., Cizadlo, T., Arndt, S., Rezai, K., Watkins, G. L., et al. (1995). Remembering the past: two facets of episodic memory explored with positron emission tomography. American Journal of Psychiatry, 152, 1576-1585.

Antrobus, J. S., Singer, J. L., Goldstein, S., \& Fortgang, M. (1970). Mindwandering and cognitive structure. Transactions of the New York Academy of Sciences, 32, 242-252.

Baker, S. C., Rogers, R. D., Owen, A. M., Frith, C. D., Dolan, R. J., Frackowiak, R. S. J., et al. (1996). Neural systems engaged by planning: a PET study of the Tower of London task. Neuropsychologia, 34, 515-526.

Bechtel, W. (2008). Mental mechanisms. London: Routledge.

Bechtel, W., \& Abrahamsen, A. (2005). Explanation: A mechanist alternative. Studies in History and Philosophy of Biological and Biomedical Sciences, 36, 421-441.

Bechtel, W., \& Abrahamsen, A. (2009). Decomposing, recomposing, and situating circadian mechanisms: Three tasks in developing mechanistic explanations. In H. Leitgeb \& A. Hieke (Eds.), Reduction and elimination in philosophy of mind and philosophy of neuroscience (pp. 173-186). Frankfurt: Ontos Verlag.

Bechtel, W., \& Abrahamsen, A. (2010). Dynamic mechanistic explanation: Computational modeling of circadian rhythms as an exemplar for cognitive science. Studies in History and Philosophy of Science Part A, 41, 321-333.

Bechtel, W., \& Abrahamsen, A. (2011). Complex biological mechanisms: Cyclic, oscillatory, and autonomous. In C. A. Hooker (Ed.), Philosophy of complex systems. Handbook of the philosophy of science, Volume 10. New York: Elsevier.

Bechtel, W., \& Richardson, R. C. (1993/2010). Discovering complexity: Decomposition and localization as strategies in scientific research. Cambridge, MA: MIT Press. 1993 edition published by Princeton University Press.

Berger, H. (1929). Über daas Elektroenkephalogramm des Menschen. Archiv für Psychiatrie und Nervenkrankheiten, 87.

Berger, H. (1930). Über daas Elektroenkephalogramm des Menschen. Zweite Mitteilung. Journal für Psychologie und Neurologie, 40.

Biswal, B., Yetkin, F. Z., Haughton, V. M., \& Hyde, J. S. (1995). Functional connectivity in the motor cortex of resting human brain using echo-planar MRI. Magnetic Resonance in Medicine, 34, 537-541.

Bremer, F. (1958). Cerebral and cerebellar potentials. Physiological Reviews, 38, 357-388.

Brown, T. G. (1914). On the nature of the fundamental activity of the nervous centres; together with an analysis of the conditioning of rhythmic activity in progression, and a theory of the evolution of function in the nervous system. The Journal of Physiology, 48, 18-46.

Buckner, R. L., Andrews-Hanna, J. R., \& Schacter, D. L. (2008). The Brain's default network. Annals of the New York Academy of Sciences, 1124, 1-38.

Buckner, R. L., \& Carroll, D. C. (2007). Self-projection and the brain. Trends in Cognitive Sciences, 11, 49-57. 
Buzsáki, G. (2006). Rhythms of the brain. Oxford: Oxford University Press.

Buzsáki, G., \& Draguhn, A. (2004). Neuronal oscillations in cortical networks. Science, 304, 1926-1929.

Canolty, R. T., Edwards, E., Dalal, S. S., Soltani, M., Nagarajan, S. S., Kirsch, H. E., et al. (2006). High gamma power is phase-locked to theta oscillations in human neocortex. Science, 313, 1626-1628.

Caton, R. (1875). The electric currents of the brain. British Medical Journal, 2.

Chemero, A., \& Silberstein, M. (2008). After the philosophy of mind: Replacing scholasticism with science. Philosophy of Science, 75, 1-27.

Cordes, D., Haughton, V. M., Arfanakis, K., Wendt, G. J., Turski, P. A., Moritz, C. H., et al. (2000). Mapping functionally related regions of brain with functional connectivity MR imaging. American Journal of Neuroradiology, 21, 1636-1644.

Darden, L., \& Craver, C. (2002). Strategies in the interfield discovery of the mechanism of protein synthesis. Studies in History and Philosophy of Biological and Biomedical Sciences, 33, 1-28.

Destexhe, A. D., Bal, T., McCormick, D. A., \& Sejnowski, T. J. (1996). Ionic mechanisms underlying synchronized oscillations and propagating waves in a model of ferret thalamic slices. Journal of Neurophysiology, 76, 2049-2070.

Destexhe, A. D., \& Sejnowski, T. J. (2003). Interactions between membrane conductances underlying thalamocortical slow-wave oscillations. Physiological Reviews, 83, 14011453.

Eccles, J. C. (1951). Interpretation of action potentials evoked in the cerebral cortex. Electroencephalography and Clinical Neurophysiology, 3, 449-464.

Felleman, D. J., \& van Essen, D. C. (1991). Distributed hierarchical processing in the primate cerebral cortex. Cerebral Cortex, 1, 1-47.

Ferrier, D. (1876). The functions of the brain. London: Smith, Elder, and Company.

Finger, S. (1994). Origins of neuroscience. Oxford: Oxford University Press.

Flourens, J. P. M. (1824). Researches Expérimentales sur les Propriétés et les Fonctions du Système Nerveux dans les Animaux Vertébris. Paris: Crevot.

Fox, M. D., Corbetta, M., Snyder, A. Z., Vincent, J. L., \& Raichle, M. E. (2006). Spontaneous neuronal activity distinguishes human dorsal and ventral attention systems. Proceedings of the National Academy of Sciences, 103, 10046-10051.

Fox, M. D., \& Raichle, M. E. (2007). Spontaneous fluctuations in brain activity observed with functional magnetic resonance imaging. Nature Reviews Neuroscience, 8, 700-711.

Fox, M. D., Snyder, A. Z., Vincent, J. L., Corbetta, M., Van Essen, D. C., \& Raichle, M. E. (2005). The human brain is intrinsically organized into dynamic, anticorrelated functional networks. Proceedings of the National Academy of Sciences of the United States of America, 102, 9673-9678.

Fox, M. D., Snyder, A. Z., Vincent, J. L., \& Raichle, M. E. (2007). Intrinsic fluctuations within cortical systems account for intertrial variability in human behavior. Neuron, 56, 171184.

Fox, M. D., Snyder, A. Z., Zacks, J. M., \& Raichle, M. E. (2006). Coherent spontaneous activity accounts for trial-to-trial variability in human evoked brain responses. Nature Neuroscience, 9, 23-25. 
Freeman, W. J., Rogers, L. J., Holmes, M. D., \& Silbergeld, D. L. (2000). Spatial spectral analysis of human electrocorticograms including the alpha and gamma bands. Journal of Neuroscience Methods, 95, 111-121.

Fukunaga, M., Horovitz, S. G., van Gelderen, P., de Zwart, J. A., Jansma, J. M., Ikonomidou, V. N., et al. (2006). Large-amplitude, spatially correlated fluctuations in BOLD fMRI signals during extended rest and early sleep stages. Magnetic Resonance Imaging, 24, 979-992.

Ghatan, P. H., Hsieh, J. C., Wirsén-Meurling, A., Wredling, R., Eriksson, L., Stone-Elander, S., et al. (1995). Brain activation induced by the perceptual maze test: A PET study of cognitive performance. Neuroimage, 2, 112-124.

Gilbert, S. J., Dumontheil, I., Simons, J. S., Frith, C. D., \& Burgess, P. W. (2007). Comment on "Wandering minds: The default network and stimulus-independent thought". Science, $317,43 b-$.

Glennan, S. (1996). Mechanisms and the nature of causation. Erkenntnis, 44, 50-71.

Glennan, S. (2002). Rethinking mechanistic explanation. Philosophy of Science, 69, S342-S353.

Gloor, P. (1969). Hans Berger on the electroencephalogram of man. Amsterdam: Elsevier.

Gong, P., \& van Leeuwen, C. (2004). Evolution to a small-world network with chaotic units. Europhysics Letters, 67, 328-333.

Goodwin, B. C. (1965). Oscillatory behavior in enzymatic control processes. Advances in Enzyme Regulation, 3, 425-428.

Greicius, M. D., Krasnow, B., Reiss, A. L., \& Menon, V. (2003). Functional connectivity in the resting brain: A network analysis of the default mode hypothesis. Proceedings of the National Academy of Sciences of the United States of America, 100, 253-258.

Greicius, M. D., \& Menon, V. (2004). Default-mode activity during a passive sensory task: Uncoupled from deactivation but impacting activation. Journal of Cognitive Neuroscience, 16, 1484-1492.

Greicius, M. D., Supekar, K., Menon, V., \& Dougherty, R. F. (2009). Resting-state functional connectivity reflects structural connectivity in the default mode network. Cerebral Cortex, 19, 72-78.

Grillner, S. (2003). The motor infrastructure: from ion channels to neuronal networks. Nature Reviews Neuroscience, 4, 573-586.

Gusnard, D. A., Akbudak, E., Shulman, G. L., \& Raichle, M. E. (2001). Medial prefrontal cortex and self-referential mental activity: Relation to a default mode of brain function.

Proceedings of the National Academy of Sciences of the United States of America, 98, 4259-4264.

Hahn, B., Ross, T. J., \& Stein, E. A. (2007). Cingulate activation increases dynamically with response speed under stimulus unpredictability. Cerebral Cortex, 17, 1664-1671.

He, B. J., Snyder, A. Z., Zempel, J. M., Smyth, M. D., \& Raichle, M. E. (2008). Electrophysiological correlates of the brain's intrinsic large-scale functional architecture. Proceedings of the National Academy of Sciences, 105, 16039-16044.

Henschen, S. E. (1893). On the visual path and centre. Brain, 16, 170-180.

Herrmann, C. S., Grigutsch, M., \& Busch, N. A. (2005). EEG oscillations and wavelet analysis. In T. Handy (Ed.), Event-related potentials: a methods handbook (pp. 229-259). Cambridge, MA: MIT.

Hille, B. (2001). Ion channels of excitable membranes. Sunderland, MA: Sinauer. 
Hodgkin, A. L., \& Huxley, A. F. (1952). A quantitative description of membrane current and its application to the conduction and excitation of nerve. Journal of Physiology, 117, 500544.

Holmes, G. M. (1918). Disturbances of visual orientation. The British Journal of Ophthalmology, 2, 449-468.

Hubel, D. H., \& Wiesel, T. N. (1962). Receptive fields, binocular interaction and functional architecture in the cat's visual cortex. Journal of Physiology, 160, 106-154.

Hubel, D. H., \& Wiesel, T. N. (1968). Receptive fields and functional architecture of monkey striate cortex. Journal of Physiology, 195, 215-243.

Huguenard, J. R. (1996). Low-threshold calcium currents in central nervous system neurons. Annual Review of Physiology, 58, 329-348.

Ingvar, D. H. (1975). Patterns of brain activity revealed by measurements of regional cerebral blood flow. In D. H. Ingvar \& N. A. Lassen (Eds.), Brain work: The coupling of function, metabolism, and blood flow in the brain: Proceedings of the Alfred Benzon Symposium VIII, Copenhagen, 26-30 May 1974, held at the premises of the Royal Danish Academy of Sciences and Letters, Copenhagen (pp. 397-413). New York: Academic.

Inouye, T. (1909). Die Sehstörungen bei Schussverletzungen der kortikalen Sehsphäre nach Beobachtungen an Verwundeten der letzten japanischen Kriege. Leipzig: Engelmann.

Jahnsen, H., \& Llinás, R. R. (1984). Electrophysiological properties of guinea-pig thalamic neurones: an in vitro study. The Journal of Physiology, 349, 205-226.

Jasper, H. H., \& Andrews, H. L. (1938). Brain potentials and voluntary muslce activity in man. Journal of Neurophysiology, 1, 87-100.

Kaada, B. R. (1953). Electrical activity of the brain. Annual Review of Physiology, 15, 39-62.

Kandel, E. R. (1976). Cellular basis of behavior: An introduction to behavioral neurobiology. San Francisco: W. H. Freeman.

Kuffler, S. W. (1953). Discharge patterns and functional organization of mammalian retina. Journal of Neurophysiology, 16, 37-68.

Kutas, M., \& Hillyard, S. A. (1980). Reading senseless sentences: brain potentials reflect semantic incongruity. Science, 207, 203-205.

Lakatos, P., Shah, A. S., Knuth, K. H., Ulbert, I., Karmos, G., \& Schroeder, C. E. (2005). An oscillatory hierarchy controlling neuronal excitability and stimulus processing in the auditory cortex. Journal of Neurophysiology, 94, 1904-1911.

Larson-Prior, L. J., Zempel, J. M., Nolan, T. S., Prior, F. W., Snyder, A. Z., \& Raichle, M. E. (2009). Cortical network functional connectivity in the descent to sleep. Proceedings of the National Academy of Sciences, 106, 4489-4494.

Lashley, K. S. (1929). Brain mechanisms and intelligence. Chicago: University of Chicago Press.

Laufs, H., Krakow, K., Sterzer, P., Eger, E., Beyerle, A., Salek-Haddadi, A., et al. (2003). Electroencephalographic signatures of attentional and cognitive default modes in spontaneous brain activity fluctuations at rest. Proceedings of the National Academy of Sciences of the United States of America, 100, 11053-11058.

Leopold, D. A., Murayama, Y., \& Logothetis, N. K. (2003). Very Slow Activity Fluctuations in Monkey Visual Cortex: Implications for Functional Brain Imaging. Cereb. Cortex, 13, 422-433. 
Leresche, N., Lightowler, S., Soltesz, I., Jassik-Gerschenfeld, D., \& Crunelli, V. (1991). Lowfrequency oscillatory activities intrinsic to rat and cat thalamocortical cells. The Journal of Physiology, 441, 155-174.

Li, C.-S. R., Yan, P., Bergquist, K. L., \& Sinha, R. (2007). Greater activation of the "default" brain regions predicts stop signal errors. Neuroimage, 38, 640-648.

Llinás, R. R. (1988). The intrinsic electrophysiological properties of mammalian neurons: Insights into central nervous system function. Science, 242, 1654-1664.

Loomis, A. L., Harvey, E. N., \& Hobart, G. A. (1937). Cerebral states during sleep, as studied by human brain potentials. Journal of Experimental Psychology, 21, 127-144.

Lorente de Nó, R. (1938). Analysis of the activity of the chains of internuncial neurons. Journal of Neurophysiology, 1, 207-244.

Machamer, P., Darden, L., \& Craver, C. F. (2000). Thinking about mechanisms. Philosophy of Science, 67, 1-25.

Mantini, D., Perrucci, M. G., Del Gratta, C., Romani, G. L., \& Corbetta, M. (2007). Electrophysiological signatures of resting state networks in the human brain. Proceedings of the National Academy of Sciences, 104, 13170-13175.

Mason, M. F., Norton, M. I., Van Horn, J. D., Wegner, D. M., Grafton, S. T., \& Macrae, C. N. (2007). Wandering Minds: The Default Network and Stimulus-Independent Thought. Science, 315, 393-395.

Mazoyer, B., Zago, L., Mellet, E., Bricogne, S., Etard, O., Houdé, O., et al. (2001). Cortical networks for working memory and executive functions sustain the conscious resting state in man. Brain Research Bulletin, 54, 287-298.

Monto, S., Palva, S., Voipio, J., \& Palva, J. M. (2008). Very Slow EEG Fluctuations Predict the Dynamics of Stimulus Detection and Oscillation Amplitudes in Humans. J. Neurosci., 28, 8268-8272.

Petersen, S. E., Fox, P. T., Posner, M. I., Mintun, M., \& Raichle, M. E. (1989). Positron emission tomographic studies of the processing single words. Journal of Cognitive Neuroscience, 1, 153-170.

Planck, M. (1949). Scientific autobiography, and other papers (F. Gaynor, Trans.). New York: Philosophical Library.

Posner, M. I., \& Raichle, M. E. (1994). Images of Mind. San Francisco: Freeman.

Raichle, M. E., MacLeod, A. M., Snyder, A. Z., Powers, W. J., Gusnard, D. A., \& Shulman, G. L. (2001). A default mode of brain function. Proceedings of the National Academy of Sciences of the United States of America, 98, 676-682.

Raichle, M. E., \& Mintun, M. A. (2006). Brain work and brain imaging. Annual Review of Neuroscience, 29, 449-476.

Raichle, M. E., \& Snyder, A. Z. (2007). A default mode of brain function: A brief history of an evolving idea. Neuroimage, 37, 1083-1090.

Rubinov, M., Sporns, O., van Leeuwen, C., \& Breakspear, M. (2009). Symbiotic relationship between brain structure and dynamics. BMC Neuroscience, 10, 55.

Shaw, J. C. (2003). The brain's alpha rhythms and the mind: A review of classical and modern studies of the alpha rhythm component of the Electroencephalogram with commentaries on associated neuroscience and neuropsychology. Amsterdam: Elsevier Publishers B.V.

Sherrington, C. S. (1913). Further observations on the production of reflex stepping by combination of reflex excitation with reflex inhibition. The Journal of Physiology, 47, 196-214. 
Sherrington, C. S. (1923). The integrative action of the nervous system. New Haven: Yale University Press.

Shulman, G. L., Fiez, J. A., Corbetta, M., Buckner, R. L., Miezin, F. M., Raichle, M. E., et al. (1997). Common blood flow changes across visual tasks. II. Decreases in cerebral cortex. [Article]. Journal of Cognitive Neuroscience, 9, 648-663.

Silber, M. H., Ancoli-Israel, S., Bonnet, M. H., Chokroverty, S., Grigg-Damberger, M. M., Hirshkowitz, M., et al. (2007). The visual scoring of sleep in adults. Journal of Clinical Sleep Medicine, 3, 121-131.

Sokoloff, L., Mangold, R., Wechsler, R. L., Kennedy, C., \& Kety, S. S. (1955). The effect of mental arithmetic on cerebral circulation and metabolism. Journal of Clinical Investigation, 34, 1101-1108.

Sporns, O., \& Zwi, J. D. (2004). The small world of the cerebral cortex. Neuroinformatics, 2, $145-162$.

Stuart, D. G., \& Hultborn, H. (2008). Thomas Graham Brown (1882-1965), Anders Lundberg (1920-), and the neural control of stepping. Brain Research Reviews, 59, 74-95.

Thagard, P. (2003). Pathways to biomedical discovery. Philosophy of Science, 70, 235-254.

Tsodyks, M., Kenet, T., Grinvald, A., \& Arieli, A. (1999). Linking spontaneous activity of single cortical neurons and the underlying functional architecture. Science, 286, 1943-1946.

van den Heuvel, M. P., Mandl, R. C. W., Kahn, R. S., \& Pol, H. E. H. (2009). Functionally linked resting-state networks reflect the underlying structural connectivity architecture of the human brain. Human Brain Mapping, 30, 3127-3141.

van Essen, D. C., \& Gallant, J. L. (1994). Neural mechanisms of form and motion processing in the primate visual system. Neuron, 13, 1-10.

Vincent, J. L., Patel, G. H., Fox, M. D., Snyder, A. Z., Baker, J. T., Van Essen, D. C., et al. (2007). Intrinsic functional architecture in the anaesthetized monkey brain. Nature, 447, 83-86.

Vincent, J. L., Snyder, A. Z., Fox, M. D., Shannon, B. J., Andrews, J. R., Raichle, M. E., et al. (2006). Coherent spontaneous activity identifies a hippocampal-parietal memory network. Journal of Neurophysiology, 96, 3517-3531.

Walter, W. G. (1936). The location of cerebral tumours by electro-encephalography. The Lancet, 228, 305-308.

Walter, W. G., \& Dovey, V. J. (1944). Electro-encephalography in cases of sub-cortical tumour. Journal of Neurology, Neurosurgery and Psychiatry, 7, 57-65.

Watts, D., \& Strogratz, S. (1998). Collective dynamics of small worlds. Nature, 393.

White, J. G., Southgate, E., Thomson, J. N., \& Brenner, S. (1986). The structure of the nervous system of the nematode Caenorhabditis elegans. Philosophical Transactions of the Royal Society of London. B, Biological Sciences, 314, 1-340.

Wilson, D. M., \& Wyman, R. J. (1965). Motor output patterns during random and rhythmic stimulation of locust thoracic ganglia. Biophysical Journal, 5, 121-143.

Wimsatt, W. C. (1976). Reductive explanation: A functional account. In R. S. Cohen, C. A. Hooker, A. C. Michalos \& J. van Evra (Eds.), PSA-1974 (pp. 671-710). Dordrecht: Reidel.

Wimsatt, W. C. (2007). Re-engineering philosophy for limited beings: Piecewise approximations to reality. Cambridge, MA: Harvard University Press. 